\title{
The Role of the Primary Visual Cortex in Perceptual Suppression of Salient Visual Stimuli
}

\author{
Georgios A. Keliris, ${ }^{1}$ Nikos K. Logothetis, ${ }^{1,3}$ and Andreas S. Tolias ${ }^{1,2,4,5}$ \\ ${ }^{1}$ Max Planck Institute for Biological Cybernetics, 72076 Tübingen, Germany, ${ }^{2}$ Department of Neuroscience, Baylor College of Medicine, Houston, Texas 77030 , \\ ${ }^{3}$ Division of Imaging Science and Biomedical Engineering, University of Manchester, Manchester M13 9PT, United Kingdom, ${ }^{4}$ Michael E. DeBakey Veterans Affairs \\ Medical Center, Houston, Texas 77030, and ${ }^{5}$ Department of Computational and Applied Mathematics, Rice University, Houston, Texas 77005
}

The role of primary visual cortex (area V1) in subjective perception has intrigued students of vision for decades. Specifically, the extent to which the activity of different types of cells (monocular versus binocular) and electrophysiological signals (i.e., local field potentials versus spiking activity) reflect perception is still debated. To address these questions we recorded from area V1 of the macaque using tetrodes during the paradigm of binocular flash suppression, where incongruent images presented dichoptically compete for perceptual dominance. We found that the activity of a minority $(20 \%)$ of neurons reflect the perceived visual stimulus and these cells exhibited perceptual modulations substantially weaker compared with their sensory modulation induced by congruent stimuli. Importantly, perceptual modulations were found equally often for monocular and binocular cells, demonstrating that perceptual competition in V1 involves mechanisms across both types of neurons. The power of the local field potential (LFP) also showed moderate perceptual modulations with similar percentages of sites showing significant effects across frequency bands (18-22\%). The possibility remains that perception may be strongly reflected in more elaborate aspects of activity in V1 circuits (e.g., specific neuronal subtypes) or perceptual states might have a modulatory role on more intricate aspects of V1 firing patterns (e.g., synchronization), not necessarily altering the firing rates of single cells or the LFP power dramatically.

\section{Introduction}

The use of visual stimuli that induce bistable perception has been established as a classical paradigm to identify the neural circuits subserving subjective perception (Attneave, 1971; Rock et al., 1994; Logothetis, 1999). A celebrated example of such a perceptual phenomenon is binocular rivalry (BR), involving alternations of visual perception between two different images presented dichoptically at corresponding retinal locations (DuTour, 1760; Wheatstone, 1838; Breese, 1899, 1909).

The primary visual cortex (V1) was implicated as an important candidate for the site of perceptual suppression during BR based on numerous psychophysical studies (Abadi, 1976; Cogan, 1987; Blake, 1989; Blake et al., 2006). In particular, competition between monocular channels offered a straightforward mechanism for the suppression of one of the two stimuli (Lehky, 1988). However, neurophysiological results in monkeys did not corroborate this hypothesis, but instead provided evidence for competition primarily between neurons in areas beyond $\mathrm{V} 1$ and

\footnotetext{
Received Feb. 8, 2010; revised June 9, 2010; accepted July 11, 2010.

This work was supported by the Max Planck Society, the National Eye Institute- National Institutes of Health (R01 EY018847), the U.S. Department of Defense (W81XWH-08-2-0147), a Merit Award from the Department of Veterans Affairs, and an Arnold and Mabel Beckman Foundation Young Investigator Award to A.S.T. We thank Dr. Theofanis Panagiotaropoulos for helping in some of the experiments and Philipp Berens for comments on a previous version of this manuscript.

Correspondence and requests for materials should be addressed to either of the following: Georgios A. Keliris, Max Planck Institute for Biological Cybernetics, 72076 Tübingen, Germany, E-mail: georgios.keliris@tuebingen. mpg.de; or Andreas S. Tolias, Department of Neuroscience, Baylor College of Medicine, Houston, TX 77030, E-mail: atolias@cns.bcm.edu.

DOI:10.1523/JNEUROSCI.0677-10.2010

Copyright $\odot 2010$ the authors $\quad 0270-6474 / 10 / 3012353-13 \$ 15.00 / 0$
}

specifically areas V4, V5/MT and IT (Logothetis and Schall, 1989; Leopold and Logothetis, 1996; Sheinberg and Logothetis, 1997). Moreover, a set of intriguing human psychophysical results conjectured that BR may involve competition between alternative higher-level stimulus perceptual interpretations (Diaz-Caneja, 1928; Kovács et al., 1996; Logothetis et al., 1996) similar to other bistable stimuli (e.g., necker cube) that do not involve interocular competition (Blake and Logothetis, 2002). Yet, subsequent studies using functional magnetic resonance imaging (fMRI) in humans, provided evidence that activity in V1 is robustly modulated by the subjective percept supporting the ocular competition hypothesis (Polonsky et al., 2000; Tong and Engel, 2001). These results engendered an apparent controversy between human fMRI and monkey electrophysiological recordings. Given the relationship between the BOLD signal and the LFP (Logothetis et al., 2001; Goense and Logothetis, 2008), one could speculate that the robust perceptual modulations reported in human fMRI studies may also be reflected in LFP signals. To this end, recent studies using the paradigm of generalized flash suppression (GFS) implicated that low-frequency LFPs show stronger modulations with perception compared with single-unit activity and high-frequency LFP (Wilke et al., 2006; Maier et al., 2008). Importantly though, GFS does not involve interocular competition like BR.

Here we undertook a study to characterize in detail and compare the extent to which different electrophysiological signals (spiking activity and various LFP frequency bands) are modulated by perception under conditions of interocular competition. We also tested directly the conjecture that monocular neurons in 
V1 robustly reflect perception (Lehky, 1988; Blake, 1989; Tong and Engel, 2001; Haynes and Rees, 2005). We used a variant of BR, namely binocular flash suppression (BFS) (Wolfe, 1984), while recording neural activity from V1 using tetrodes. The first electrophysiological studies using BFS were performed in anesthetized cats (Sengpiel and Blakemore, 1994; Sengpiel et al., 1995) and implicated that interocular interactions at the level of binocular neurons in V1 could provide a possible neural basis for the perceptual switches experienced during BR. Later on, BFS paradigms in awake, behaving monkeys as well as humans have been successfully used in electrophysiological experiments to study the role of higher areas in subjective perception (Sheinberg and Logothetis, 1997; Kreiman et al., 2002; Maier et al., 2007).

In our study, we recorded spiking activity from hundreds of single units and simultaneously acquired LFP signals during the dichoptic presentation of orthogonal sinusoidal gratings. We find the following. (1) In agreement with previous studies (Leopold and Logothetis, 1996) only a moderate percentage of neurons $(20 \%)$ in V1 is modulated in parallel with perception. The magnitude of their modulation is substantially smaller than the physical preference of these neurons. (2) Neurons showing perceptual modulations in V1 are from both binocular and monocular classes with equal probability. (3) Only moderate perceptual modulations of the power in different frequency bands of the LFP are found.

\section{Materials and Methods}

Electrophysiological recordings and surgical methods. Electrophysiological recordings were performed in two healthy adult male rhesus monkeys (Macaca mulatta) weighing 16 and $11 \mathrm{~kg}$ respectively (monkeys D98 and F03). All experiments were conducted with great care to ensure the well being of the animals and they were in full compliance with the guidelines of the local authorities (Regierungspräsidium) and the European Community (EUVD 6/609/EEC) for the care and use of laboratory animals. Recording chambers were positioned stereotactically over the operculum in area V1 in three hemispheres (both hemispheres in D98 and right hemisphere of F03) with the aid of high-resolution magnetic resonance anatomical imaging. These images were collected in a vertical 4.7 tesla scanner with a $40 \mathrm{~cm}$ bore-diameter (Biospec 47/40c; Bruker Medical). The system had a $50 \mathrm{mT} / \mathrm{m}(180 \mu$ s rise time $)$ actively shielded gradient coil (B-GA 26, Bruker Medical) of $26 \mathrm{~cm}$ diameter. A custom chair and custom system for positioning the monkeys in the magnet were used. We collected anatomical data using T1-weighted high resolution $(256 \times$ $256 \times 160$ real data points at $0.5 \mathrm{~mm}$ isotropic linear resolution) images with 3D-MDEFT (modified driven equilibrium Fourier transform) pulse sequences, with an echo time (TE) of $4 \mathrm{~ms}$, repetition time (TR) of $22 \mathrm{~ms}$, flip angle (FA) of $20^{\circ}$ and four segments. The anatomical scans were done while the animals were under general anesthesia. The skull parameters were extracted using morphological methods (Paravision; Bruker Medical) and we created a 3D rendered surface (Analyze; Mayo Foundation, Rochester, NY) for designing the cranial headpost and the recording chambers to fit the skull surface. A 5-axis CNC machine (WilleminMacodel W428) was used to build these form-specific implants that resulted in an excellent fit between the implants and the underlying skull surface. These methods have been described in detail previously (Logothetis et al., 1999).

An array of tetrodes was chronically implanted over the operculum in area V1 inside a form-specific chamber constructed from medical-grade titanium (monkey D98 left hemisphere). In both monkeys, we also recorded nonchronically from form-specific chambers implanted in the right hemispheres. The chamber of monkey D98 was made of medicalgrade titanium while the chamber of monkey F03 was made of polyether ether ketone (TECAPEEK; Ensinger GmbH). All chambers were implanted under aseptic conditions under general anesthesia. Initially, the animals received subcutaneous injections of Rubinol $(0.01 \mathrm{mg} / \mathrm{kg})$ and Ketavet $(15 \mathrm{mg} / \mathrm{kg})$ and subsequently they were prepared for intubation by intravenous injections of fentanyl $(0.003 \mathrm{mg} / \mathrm{kg})$, trapanal $(5 \mathrm{mg} / \mathrm{kg})$ and lysthenol $(3 \mathrm{mg} / \mathrm{kg})$. During surgery, the animals received balanced anesthesia consisting of isoflurane $1.3 \%$. The surgical procedures are described in detail previously (Logothetis et al., 2002). All recordings were conducted with tetrodes attached to microdrives that could be manually adjusted independently. For the chronic recordings, neural activity was recorded using a custom-built array of tetrodes (Tolias et al., 2007). The distance between nearby tetrodes was $200 \mu \mathrm{m}$. For the nonchronic recordings, one to four (two in most sessions) manually adjustable microdrives (Crist Instrument Co.) were inserted into a custom-built grid and activity was recorded using tetrodes.

Multiunit and single-unit activity was sampled at $32 \mathrm{kHz}$, digitized (12 bits), and stored using the Cheetah data acquisition system (Neuralynx). LFP signals were recorded by filtering the raw voltage signal using analog bandpass filtering (high-pass set at $1 \mathrm{~Hz}$ and low-pass set at $475 \mathrm{~Hz}$ ) and digitized at $2 \mathrm{kHz}$ (12 bits). Multiunit activity was defined as the events that exceeded a predefined threshold $(25 \mu \mathrm{V})$ of the filtered, digitized signal (analog filtering high-pass set at $600 \mathrm{~Hz}$ and low-pass set at $6 \mathrm{kHz}$ and digitized at $32 \mathrm{kHz}, 12$ bits). Single units were isolated using a custom-built offline clustering system working on features extracted from the recorded waveforms (Tolias et al., 2007). No preselection functional criteria were applied for the neurons. Details of single-unit isolation methods have been described previously (Tolias et al., 2007).

The animals were implanted with a scleral search coil (Robinson, 1963; Judge et al., 1980) and their eye movements were monitored on-line. Data were also collected for off-line analysis using both the QNX-based data acquisition system at $200 \mathrm{~Hz}$ and the Cheetah data acquisition system at $2000 \mathrm{~Hz}$.

Visual stimulation and behavioral paradigm. Visual stimuli were displayed using a dedicated graphics workstation (TDZ 2000; Intergraph Systems) running an OpenGL-based stimulation program. Stimuli were presented dichoptically by using a custom-made stereoscope with two LCD monitors at both sides running at a resolution of $1280 \times 1024$ and a refresh rate of $60 \mathrm{~Hz}$. The behavioral aspects of the experiment were controlled using the QNX real-time operating system (QNX Software Systems Ltd).

At the beginning of each session, the mirror stereoscope was positioned in front of the monkeys' head and two circular apertures were aligned with the animal's eyes. These apertures served to limit the visual field to the central $15^{\circ}$ of visual angle and prevented nasal viewing of the opposite display. In succession, we calibrated the monkeys' eye movements using a fixation-saccade task. To ensure that the two displays were correctly aligned in front of the two eyes we used the following procedure. First we calibrated the left eye alone while the right eye display was kept blank. The animal had to fixate briefly on a central fixation spot $\left(0.2^{\circ}\right)$ and then saccade to a second fixation target switched on at its periphery randomly in eight different directions $\left(\Delta \varphi=45^{\circ}\right)$. After successful acquisition of the presented saccadic locations, the amplitude of the saccades was increased until the monkey failed, in this way mapping exactly the visible portion of the monitor. When the first eye was fully calibrated we switched to an iterative procedure between the two eyes: first the central fixation spot was presented briefly to the left eye. After the monkey acquired fixation, it was switched off and switched on in the right eye. If the monkey could not fuse (i.e., directly overlay the two stimuli) he performed a saccade to a new location. The monitoring system estimated the amplitude of his saccade by calculating the difference between the two fixations and moved the location of the right eye fixation in the opposite direction for the following trial. Usually after a few trials of calibration (typically four to five) the monkeys were able to fixate the target continuously (although it was switched between the eyes) and we concluded that they could correctly overlay (fuse) the two displays. The fixation-saccade procedure was then performed for the right eye alone with all the targets displaced according to the offset values registered in the previous eye-switching procedure. Finally, we checked the calibration with both displays on. The full calibration described above was performed additionally at the end of the experiments and sometimes between the sessions to ascertain that the two displays remained correctly aligned. During calibration, the monkey received a drop of juice at end of each trial. 
A

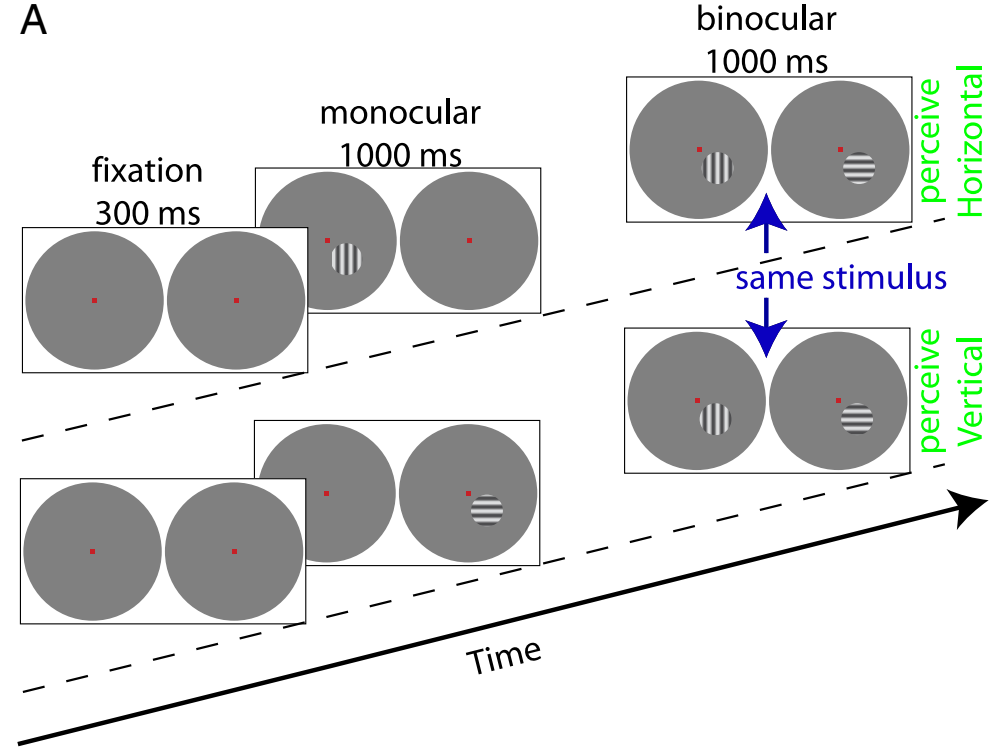

B

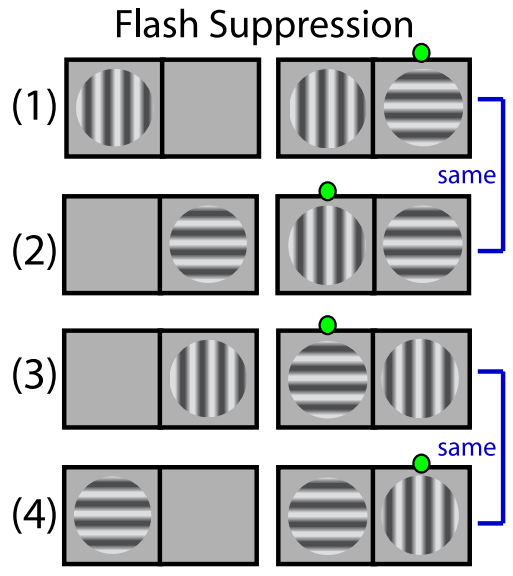

Conditions

(5)

Physical Alternation

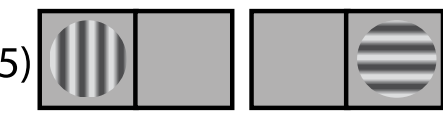

(6)

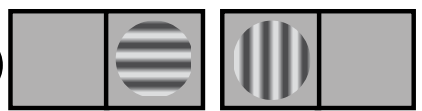

(7)
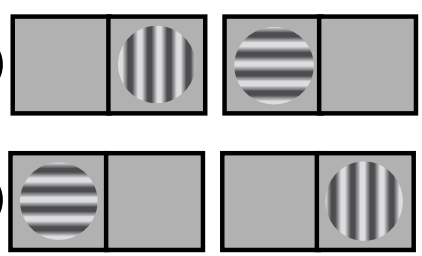

Figure 1. Illustration of the binocular flash suppression (BFS) behavioral paradigm. $A$, An example of two conditions for which the subject experiences different percepts (a horizontal or a vertical grating) although the exact same stimuli are shown in the two displays during the binocular period of presentation. In each condition, the subjects had to initially fixate a small red target at the center of the display for $300 \mathrm{~ms}$ and then a circular sinusoidal grating stimulus was presented parafoveally for 1 s in one of the two eyes. In succession, a grating with orthogonal orientation to the initially presented one was shown in a corresponding location in the opposite eye while the first grating remained on the display for another second. During this binocular presentation period, the newly appearing grating is invariably perceived while the simultaneously presented grating in the opposite eye is extinguished from perception. $\boldsymbol{B}$, Battery of conditions used in our experiments. Note that in different sessions we have used different pairs of orthogonal orientations, not only horizontal and vertical as depicted here. The pair of conditions described above in $\boldsymbol{A}$ are depicted here in the diagrams $1-2$. They compose one of two stimulus configurations that allow comparison of the exact same stimuli (which lead to different percepts) during the binocular presentation period. In the second configuration (diagrams 3-4), identical stimuli are presented with reversed eye of presentation. Each BFS condition (1-4) can also be compared with a "control" physical alternation condition (5-8), which demonstrates an identical perceptual experience without binocular conflict. In these conditions, the first grating is simply removed on presentation of the second. In each diagram, the leftward pair of displays represents the monocular period and the rightward the binocular. The green dots denote the grating that was perceived during the binocular period.

After eye calibration and alignment of the displays, a coarse receptive field mapping was performed to position the stimulus for the experiments. Oriented gratings (similar to the ones used in the BFS experiments) were presented parafoveally while the monkey fixated a central target for 2-3 s. These gratings were presented binocularly and their position, size and orientation could be changed manually while the monkey kept fixating the central target. The multiunit responses of the cells were put through a sound amplifier (Grass Technologies) so that the experimenter could evaluate the gross location of the receptive fields and the orientation preferences of the multiunit responses. The pair of orthogonal orientations exhibiting maximal differential multiunit re-

sponses was then selected to be used in the BFS experiments. We will further refer two these two orthogonal orientations as $\theta$ and $\theta_{\text {orth }}$. In a subset of experiments for which more than one tetrode was used, the stimulus optimization was typically performed separately for each site.

To study the relationship between neural activity and perception, we used the paradigm of BFS. In this case, two rivalrous patterns are presented dichoptically and asynchronously to the two eyes. Under these conditions, the latter pattern dominates perceptually over the first, provided the two differ sufficiently in their structure (Wolfe, 1984). Several studies have shown that BFS is intimately related to BR at the psychophysical level. Specifically, the results of several parametric manipulations of BFS suggested a common mechanism between $\mathrm{BR}$ and BFS. Moreover, forward masking or simple light adaptation are not thought to underlie the perceptual suppression during BFS (Wolfe, 1984; Blake et al., 1990; Baldwin et al., 1996; Kreiman and Koch, 1999; Kreiman, 2001; Brascamp et al., 2007). A recent study (Tsuchiya et al., 2006) compared the depth of suppression for BR and BFS by using a probe detection task and found that BFS induces a transient increase of suppression up to $300 \mathrm{~ms}$ after the flash but then suppression becomes at the same level as in the case of BR (to avoid this transient effects of BFS in our study we excluded the first $500 \mathrm{~ms}$ after the flash). Based on this evidence, the authors argue that the two paradigms are based on the same mechanism of perceptual suppression. Similarly, a computational modeling study (Nichols and Wilson, 2009) demonstrated that the differences between the techniques can be attributed to the sustained versus transient stimulation rather than a difference in the mechanism of perceptual suppression. Therefore, BFS is thought to involve a temporary burst of inhibition at the onset of the flash that might be critical for the perceptual switch (Macknik and Livingstone, 1998; Macknik et al., 2000) but it is certain that BR prevails after a short delay and the subjects experience spontaneous alternations.

We used the following experimental paradigm in our experiment: after the monkey acquired fixation on a colored square target $\left(0.2^{\circ}\right)$ for $300 \mathrm{~ms}$, static, sine-wave grating stimuli were presented dichoptically to the two eyes. Typically the size of the gratings was $1-2^{\circ}$ in diameter, the spatial frequency $3-5$ cycles per degree and the contrast 70\%. During BFS, a grating stimulus was displayed for $\sim 1 \mathrm{~s}$ monocularly to the left or the right eye followed by the onset of a second orthogonal grating ("the flash") to the corresponding location in the other eye for another second without removal of the first grating resulting in a binocular incongruent condition (Fig. 1). The $1 \mathrm{~s}$ initial monocular presentation reliably biases the perception toward the second grating flashed during the binocular presentation. At the end of each successful trial, a drop of apple juice was delivered to the animal as a reward. For the physical alternation condition, the trials started in the same way with a $300 \mathrm{~ms}$ fixation and a monocular presentation of a grating stimulus for $1 \mathrm{~s}$. Then, similar to the BFS trials, a second orthogonal grating was presented in the second eye but the first grating was removed upon presentation of the second (Fig. 
Table 1. Numbers and percentages of significant modulations

\begin{tabular}{lllll}
\hline & & SUA & LFP (4-20 Hz) & LFP (30-90 Hz) \\
\hline T & Total \# of isolated single units/recorded sites & 582 & 381 & 381 \\
VR & Visually responsive (\% of T) & $523(90 \%)$ & $362(95 \%)$ & $362(95 \%)$ \\
SM & Sensory stimulus modulation (\% VR) & $371(71 \%)$ & $149(41 \%)$ & $275(76 \%)$ \\
PM & Perceptual stimulus modulation (\% VR) & $104(20 \%)$ & $79(22 \%)$ & $54(18 \%)$ \\
PaS & Perceptual \& sensory (\% PM) & $94(90 \%)$ & $55(70 \%)$ & $24(86 \%)$ \\
XP & Only perceptual (\% PM) & $10(10 \%)$ & $24(30 \%)$ & $9(14 \%)$ \\
\hline
\end{tabular}

The absolute numbers and respective percentages of significant modulations are presented for single units [SUA (single-unit activity)] and two frequency bands of the local field potential: $4-20 \mathrm{~Hz}$ and $30-90 \mathrm{~Hz}$. In the first row ( $\mathrm{T}$ ) the total numbers of isolated single cells and recorded LFP sites are reported. The second row (VR) presents the number (percentage) of cells/sites that showed significant visual responses. The third row (SM) presents the number of cell/sites that were responding differentially two the different congruent stimuli (sensory modulation) and the fourth row (PM) the number of cells/sites that showed differential responses under the different perceptual conditions (under the same stimulus) as a percentage of visually responsive cells/sites. In the last two rows: PaS presents the numbers of perceptually modulating cells/sites that showed, in addition, sensory modulations and $\mathrm{xP}$ presents the numbers of cells/sites that showed exclusively perceptual modulations.

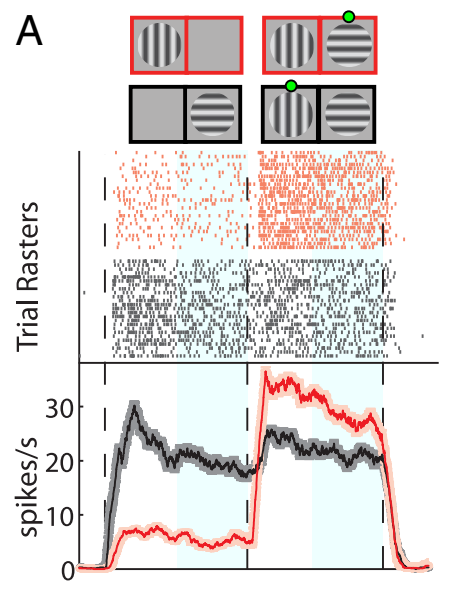

D

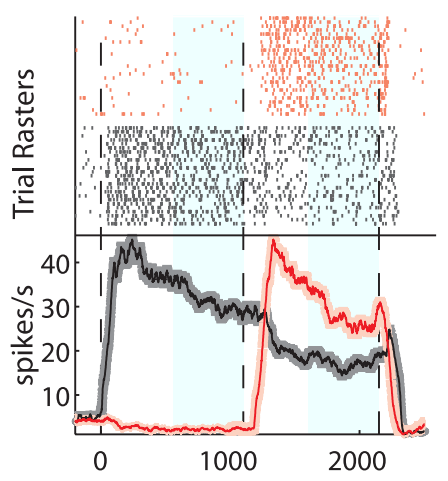

B

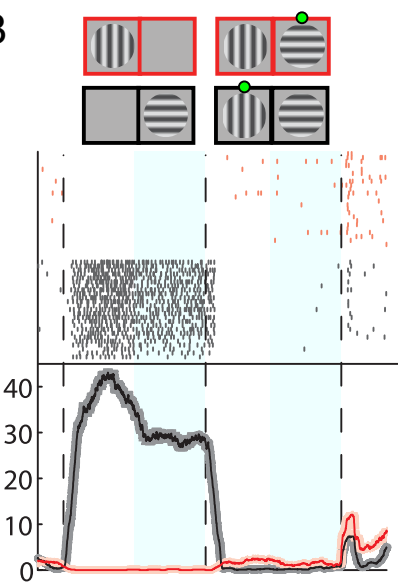

E

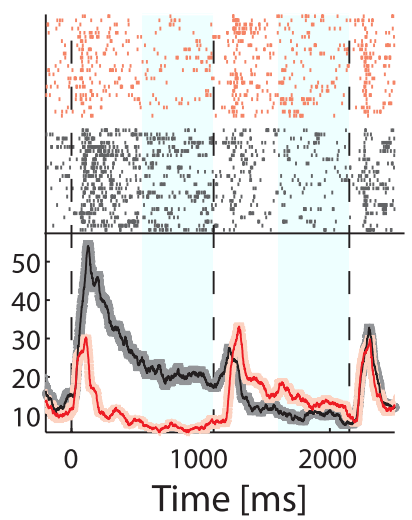

C

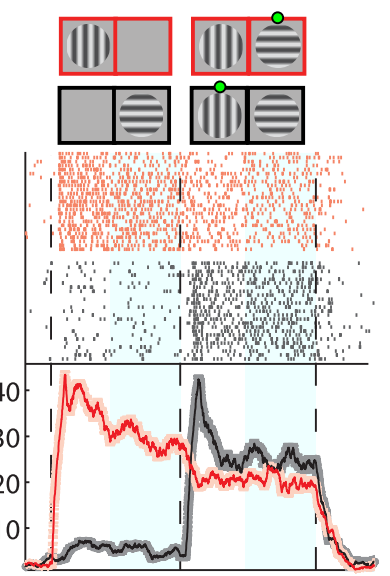

$\mathrm{F}$

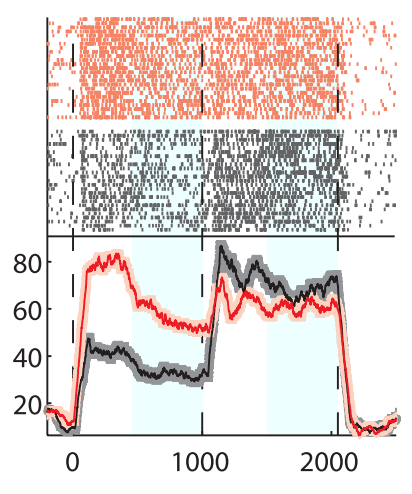

Figure 2. Examples of single-unit modulations during BFS. $\boldsymbol{A}-\boldsymbol{F}$, In each panel, the activity of one single neuron is presented. In the first three panels $(\boldsymbol{A}-\boldsymbol{C}$, neurons from monkey $\mathrm{D} 98$ are shown and in the last three $(\boldsymbol{D}-\boldsymbol{F})$, neurons from monkey $\mathrm{F} 03$. The diagrams on the top of the panels demonstrate the sequence of stimulus presentation with a green dot denoting the percept during the binocular period (1000-2000 ms). Note that in general we used different pairs of orthogonal gratings (see Materials and Methods) but here cases where the monkey was presented with a horizontal-vertical pair are shown. In each panel, raster plots of 25 trials (rows) of each of two conditions with the same stimulation but different perceptual outcome (see Fig. 1 and Materials and Methods) are presented in the upper part in red and dark gray. At the lower part, the corresponding spike-density-functions (red and dark gray lines) are presented. Spike-density-functions were calculated using a convolution of the spike-trains with a rectangular window of $100 \mathrm{~ms}$ width. The shaded areas (lighter red and gray) represent SEM from a total of 100 trials per neuron. Light blue shadings at the background between 500 and 1000 and 1500 and 2000 ms denote the time windows for which we performed the statistical comparisons.

$1 B)$. Importantly, this resulted in no binocular incongruence but to a simple successive presentation of the two orthogonal orientation gratings in opposite eyes. These conditions mimic the perceptual experiences of the subjects during BFS without introducing binocular conflict and therefore are termed "physical alternation." During presentation of the stimuli, the monkey had to keep fixating within a circular window with a radius of $0.5^{\circ}$ from the center of the colored fixation target; failure to do so resulted in abortion of the trials and no juice reward.

After the end of all recording sessions, one of the animals (D98) was trained to directly report its perception during the presentation of the stimuli by holding one of two levers. The left lever corresponded to the perception of one orientation $\left(135^{\circ}\right)$ while the right lever to the perception of the orthogonal $\left(45^{\circ}\right)$. During training, the animal was solely presented with physical alternation trials (i.e., without incongruence) and the two orthogonal orientations were switched at intervals drawn from a gamma distribution given by the following:

$$
f(x ; \lambda, r)=x^{r-1} \frac{e^{-\lambda x}}{\Gamma(r)} \lambda^{r},
$$

with parameters $\lambda=8.3$ and $r=7.2$ that were identical with the ones calculated for monkeys reporting binocular rivalry (Leopold and Logothetis, 
1996). Trials started with the presentation of a fixation target for $300 \mathrm{~ms}$ after which one of the stimuli (pseudorandomly selected) was presented parafoveally. At the onset of the stimulus and after every stimulus switch, the animal was expected to press the correct lever within a maximum response time of $1 \mathrm{~s}$ or else the trial was considered incorrect and aborted. After pressing the correct lever, the animal had to keep holding the lever either up to the next stimulus switch (for which it was required to change its response) or until the end of the trial which was signified with the removal of the stimulus. If the animal responded correctly and kept his gaze within a window of $\pm 0.5^{\circ}$ around the fixation target for the whole interval, the trial was considered correct and the animal received a small amount of apple juice as a reward. Correct trials ended after a minimum prespecified time (typically $4 \mathrm{~s}$ ) was crossed by the last stimulation interval, which was not interrupted until its completion (stimulation intervals were drawn from a gamma distribution as above). This resulted in trials of variable durations (depending on the duration of the last alternation period) and variable number of alternation periods. Typically, trials lasted $4-6 \mathrm{~s}$ and the animal had to report two to five stimulus switches. Importantly, in this stimulation scheme stimulus switches were unpredictable to the animal. When the animal reached a performance level of $>95 \%$ correct responses, we gradually started presenting to it a small percentage $(<25 \%)$ of incongruent stimulation trials randomly interleaved with the physical alternation trials. The incongruent stimulation trials started with a monocular presentation of one of the orthogonal gratings for $1 \mathrm{~s}$ after which the second orientation grating was switched on in the opposite eye without removal of the first grating. Note that this presentation is identical to the BFS presentation we have used during the recording sessions. During those sessions, the stimulus presentation lasted for $2 \mathrm{~s}(1 \mathrm{~s}$ monocular and $1 \mathrm{~s}$ binocular). For these experiments, the binocular stimulus presentation was extended to longer periods (as much as the minimum time of the physical alternation trials- typically $4 \mathrm{~s}$ ) in contrast to the $1 \mathrm{~s}$ used for the BFS recordings. This was intentionally designed to allow the animal to respond to potential spontaneous perceptual reversals following the induced reversals, which we expected to happen shortly after the "flash" of the second stimulus. During the binocular stimulus presentation, the animal was allowed to press any of the two levers and to change its response from one to the other lever an arbitrary number of times. Similar to the physical alternation trials, we required that it did so within a maximum interval of $1 \mathrm{~s}$, or else the trial was considered incorrect and aborted. If the animal followed the above criteria for the whole duration of the stimulation interval we considered the trial as correct and the animal was rewarded with a small amount of juice as in the physical alternation trials.

Statistical and data analysis. Custom programs written in Matlab (MathWorks) were used for data analysis. Statistical significance of sensory and perceptual modulations was assessed by using a nonparametric Wilcoxon rank sum test (also referred to as the Mann-Whitney $U$ test) that performs a two sided test of the null hypothesis that the data of two conditions are independent samples from identical continuous distributions with equal medians, against the alternative that they do not have equal medians. Since for every neuron or recording site we presented two different configurations of the stimuli according to the eye of presentation, we tested each configuration independently and then we corrected for multiple comparisons using a Bonferroni correction. We considered a neuron/site to show significant sensory/perceptual modulations when at least one of the pairs of conditions tested achieved a significance level $p$ (Bonferroni corrected) smaller than the critical significance level $\alpha(\alpha=0.05)$. For all of our comparisons, we excluded the first $500 \mathrm{~ms}$ of the responses to avoid effects biased to the initial transients. As a result, we always used the last $500 \mathrm{~ms}$ of each condition that effectively reflected the sustained part of the responses.

The preference/modulation index was computed using $d^{\prime}$ that is related to the discriminability of the two conditions and is defined as follows:

$$
d^{\prime}=\frac{\mu_{A}-\mu_{B}}{\hat{\sigma}}
$$

Here $\mu_{A}$ and $\mu_{B}$ denote the mean responses to the two conditions being compared and $\hat{\sigma}=\sqrt{\left(\hat{\sigma}_{A}^{2}+\hat{\sigma}_{B}^{2}\right) / 2}$ is the pooled variance of the two response distributions. For our purpose, $d^{\prime}$ indices were calculated either for pairs of monocularly presented orientation gratings (referred to as sensory or physical preference $d^{\prime}$ ), or under the dichoptic presentation of
Physical Alternation
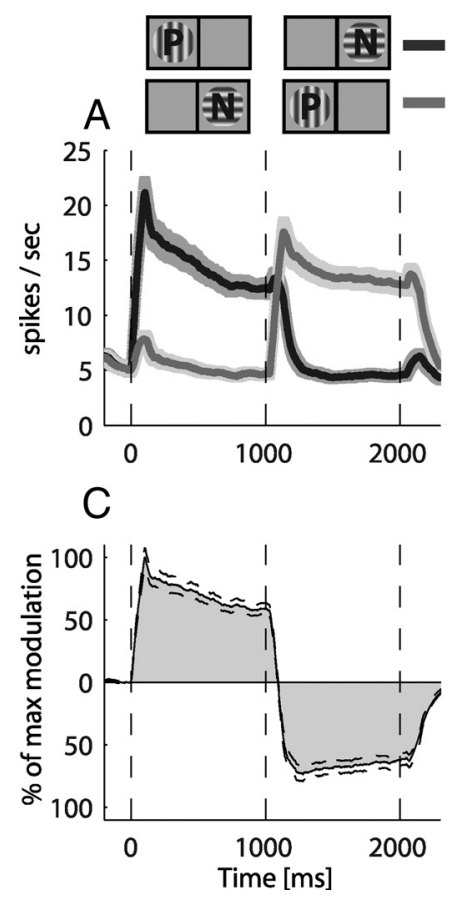

Flash Suppression
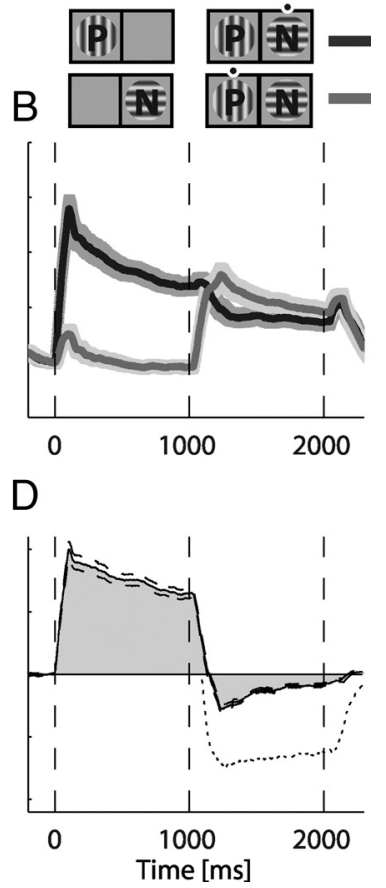

Figure 3. Population responses during physical alternation and binocular flash suppression conditions. Before averaging, conditions were sorted to preferred $(\mathrm{P})$ and nonpreferred (N) according to the responses during the monocular period of presentation. Plots in $\boldsymbol{A}$ and $\boldsymbol{B}$ depict average spike density functions for all physically selective neurons $(n=371)$. The upper part of the figure shows a diagram of the different conditions. A vertical grating is marked with $\mathbf{P}$ (preferred) and a horizontal with $\mathbf{N}$ (nonpreferred) for reasons of clarity. In essence, any one of 8 different orientations could be preferred with its orthogonal being the nonpreferred. The black dots in the binocular period denote the grating which was perceived. In $A$, the mean time courses of the population activity under the physical alternation conditions are presented. Dark solid lines represent the mean spike density functions for a presentation sequence of preferred $(0-1000 \mathrm{~ms})$ followed by the nonpreferred $(1000-2000 \mathrm{~ms})$ grating. Light gray solid lines represent the mean spike density functions for a presentation of the nonpreferred grating first, followed by the preferred. Shaded areas around the lines represent SEMs calculated over the neurons averaged. In $\boldsymbol{B}$, the mean population time courses for the binocular flash suppression conditions are plotted in a similar manner. In $\boldsymbol{C}, \boldsymbol{D}$, the average differences between the preferred and nonpreferred conditions are depicted for the same populations of cells for the physical alternation $(\boldsymbol{C})$ and flash suppression $(\boldsymbol{D})$. Modulations are expressed as a percentage of the maximum modulation during the monocular presentation $(0-1000 \mathrm{~ms})$. The dotted line in $D$ is a copy of the physical alternation modulation in C to provide a means of direct comparison with the perceptual modulation during flash suppression.

two incongruent orthogonal gratings each perceived at a time (referred to as perceptual preference $d^{\prime}$ ). Ocularity preference was calculated by comparing the monocular responses of the preferred orientation across the two eyes. We have always used right eye as condition A therefore responses were positive for right-eye selective sites and negative for left eye selective sites. The orientation preference for each cell was defined as the $d^{\prime}$ value between the two orthogonal orientations presented to the preferred eye. Similarly, perceptual modulation was defined as the $d^{\prime}$ value between the binocular incongruent conditions with the same stimuli and different percepts (Fig. 1).

The percentages of perceptual modulations in different classes of neurons were compared by using a $\chi^{2}$ test for homogeneity (also referred to as contingency table analysis) by using the following formula:

$$
\chi^{2}=\sum \sum \frac{\left(f_{i j}-\hat{f}_{i j}\right)^{2}}{\hat{f}_{i j}} .
$$

In this formula, $f_{i j}$ refers to the frequency expected in a row $i$ column $j$ if the null hypothesis (i.e., that the percentage is independent of class) is true. We analyzed the modulations across ocularity and orientation pref- 


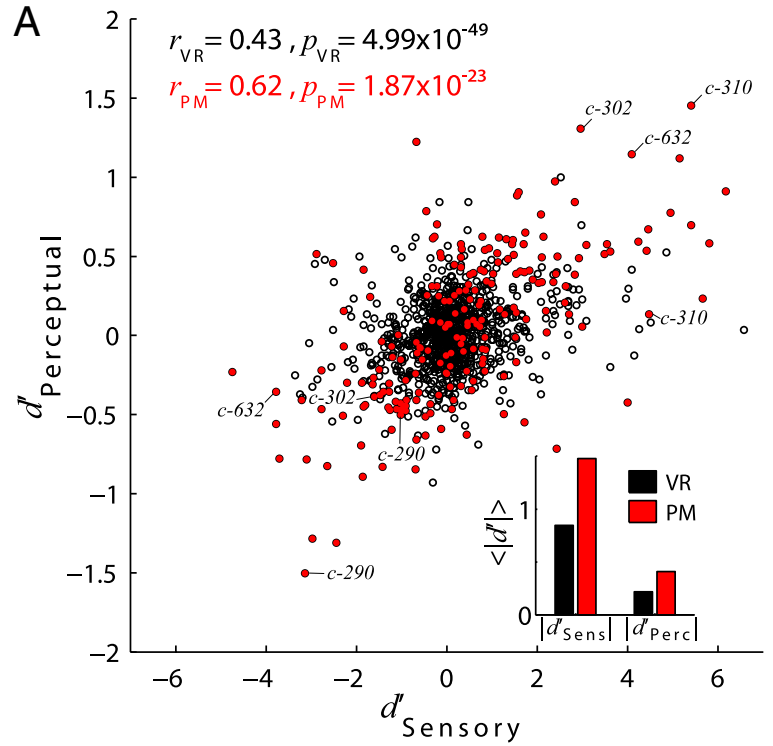

B

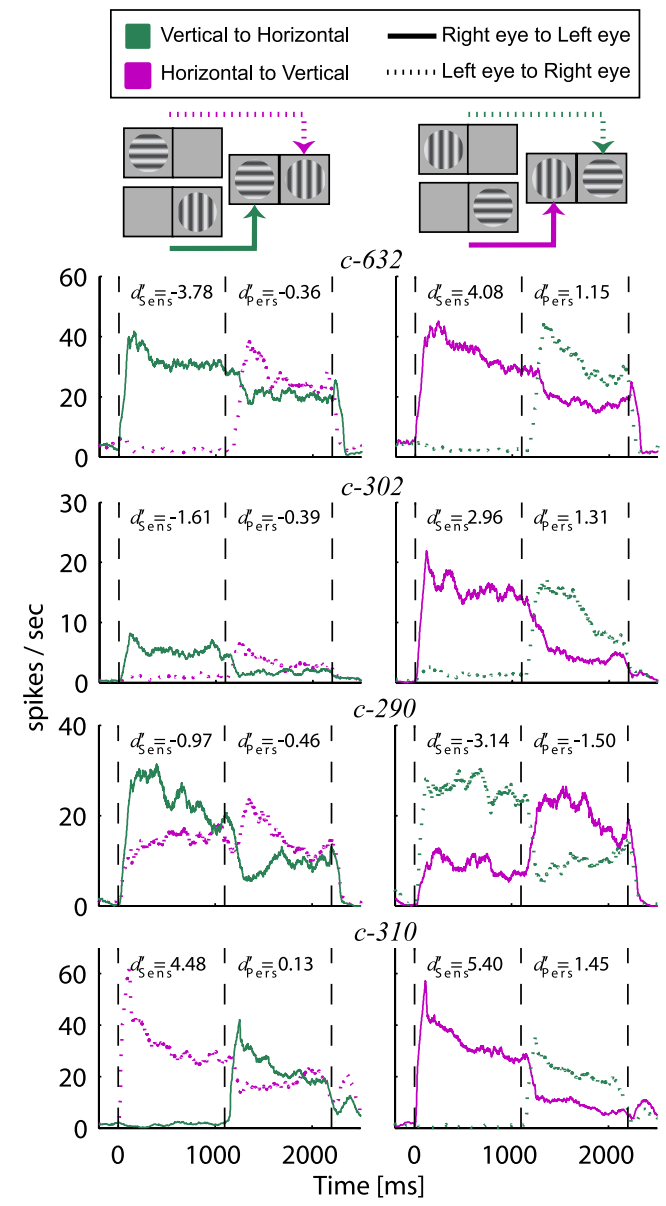

Figure 4. A, Scatter plot of sensory versus perceptual modulation of the neuronal responses. The sensory preference (time window $500-1000 \mathrm{~ms}$ ) was calculated for all visually responsive (VR) neurons ( $n=523$, black open circles) by the $d^{\prime}$ index (see Materials and Methods) between the monocular orthogonal gratings presented in the left and right eyes for each configuration. Note that this sensory preference (between orthogonal gratings in opposite eyes) can be because of orientation or ocularity preference, or a combination of both. A corresponding (according to which stimulus is perceived under BFS) perceptual preference was calculated using a $d^{\prime}$ index during the binocular presentation of the two gratings (time window 1500-2000 $\mathrm{ms})$. Positive values indicate that the response of the neuron was greater during presentation/perception of orientation $\theta$ and negative values that the response was higher during presentation/ perception of the orthogonal orientation $\theta_{\text {orth }}$. Red filled circles correspond to the neurons erence, by partitioning the data into segments containing approximately equal numbers of cells using Gessaman partitioning (Devroye et al., 1996) and then used the same $\chi^{2}$ test for homogeneity.

Eye movement analysis. We first calculated the time series of eyevelocities by differentiation of the position signals. Then, the horizontal and vertical angular velocities were independently thresholded at seven times their median-based SD to detect putative microsaccadic events. An event was classified as a microsaccade if the following additional criteria were satisfied: (1) it had a minimum duration of 8 $\mathrm{ms},(2)$ it had an amplitude between 1 and $60 \mathrm{~min}$ of a degree, and (3) it had a maximum peak-velocity of $110^{\circ}$ per second (Engbert and Kliegl, 2003). These parameters provided a fairly accurate detection of microsaccades according to visual inspection of the results (see for example supplemental Fig. $1 A, B$, available at www.jneurosci.org as supplemental material). In addition, the extracted microsaccades satisfied the main-sequence criterion and showed high correlation of amplitude and velocities (supplemental Fig. $1 D$, available at www. jneurosci.org as supplemental material). Fixation locations were extracted as the mean positions between saccades (supplemental Fig. $1 A-C$, available at www.jneurosci.org as supplemental material) and the microsaccade directions were calculated from the initial and final positions of the eye during a microsaccade event. Finally, we used the onset time of microsaccades to calculate microsaccadic rates over time. Statistical analysis was performed on a dataset by dataset basis and compared the distributions of the extracted eye-movement parameters (fixation locations, microsaccade amplitudes, microsaccade directions and microsaccade rates) under the same conditions and times as for the electrophysiological signals. A Wilcoxon-rank sum test $(\alpha=0.05)$ was used to compare the distributions of fixation locations, microsaccade amplitudes and microsaccade rates. A circular analog of the Kruskal-Wallis test and specifically the circ_cmtest function of the CircStat Matlab toolbox (Berens, 2009) was used for microsaccade directions.

Local field potential analysis. The power spectral density (PSD) of the raw LFP signals was estimated using the multitaper method (Thomson, 1982). This method uses linear or nonlinear combinations of modified periodograms to estimate the PSD. These periodograms are computed using a sequence of orthogonal tapers (windows in the frequency domain) specified from the discrete prolate spheroidal sequences. We used an adaptive nonlinear combination of seven tapers with a timebandwidth product $(\mathrm{NW}=4)$ of four for each $500 \mathrm{~ms}$ data segment. This resulted in spectra with independent frequency bins with a bandwidth of $8 \mathrm{~Hz}$. Average spectrograms were calculated by moving $500 \mathrm{~ms}$ windows with $90 \%$ overlap.

The LFP power over time was calculated by bandpass filtering the raw signal in different frequency bands. Digital filters were constructed via the Parks-McClellan optimal equiripple FIR filter design with an attenuation factor of $60 \mathrm{~dB} / \mathrm{Hz}$ outside the cutoff frequencies.

\footnotetext{
$(n=104)$ that showed significant perceptual modulations (Wilcoxon rank sum test, $\alpha=0.05$ ) in at least one of the two configurations. A Pearson correlation coefficient was calculated for the whole population of visually responsive (VR) cells as well as for the subset of perceptually modulating cells (PM) and are presented with their $p$ values at the upper left part of the plot in black and red, respectively. The inset at the lower right presents a bar plot of the mean absolute sensory [VR: $0.84 \pm 0.03$ (SEM), PM: $1.48 \pm 0.09$ (SEM)] and perceptual $d^{\prime}$ indices [VR: $0.22 \pm$ 0.01 (SEM), PM: $0.41 \pm 0.02$ (SEM)] for the two populations. $\boldsymbol{B}$, The spike density functions of four PM neurons are presented. For each neuron, responses from both stimulus configurations are presented in the left and right columns. The diagrams above each column demonstrate the stimuli used in each configuration; a horizontal grating was used to depict orientation $\theta$ and a vertical for the orthogonal $\theta_{\text {orth }}$. The perceptual transitions from $\theta$-to- $\theta_{\text {orth }}$ and $\theta_{\text {orth }}$-to- $\theta$ are depicted by magenta and green colors respectively while the transitions from right-to-left eyes or from left-to-right with solid and dotted lines, respectively. The sensory and perceptual $d^{\prime}$ are printed on the upper side of the plots for each condition compared. The cell identifications are also presented in the scatter plot in $\boldsymbol{A}$ for better visualization. Note that each cell is represented in the scatter plot with two points reflecting the two stimulus configurations from the left and right columns of $\boldsymbol{B}$.
} 
A
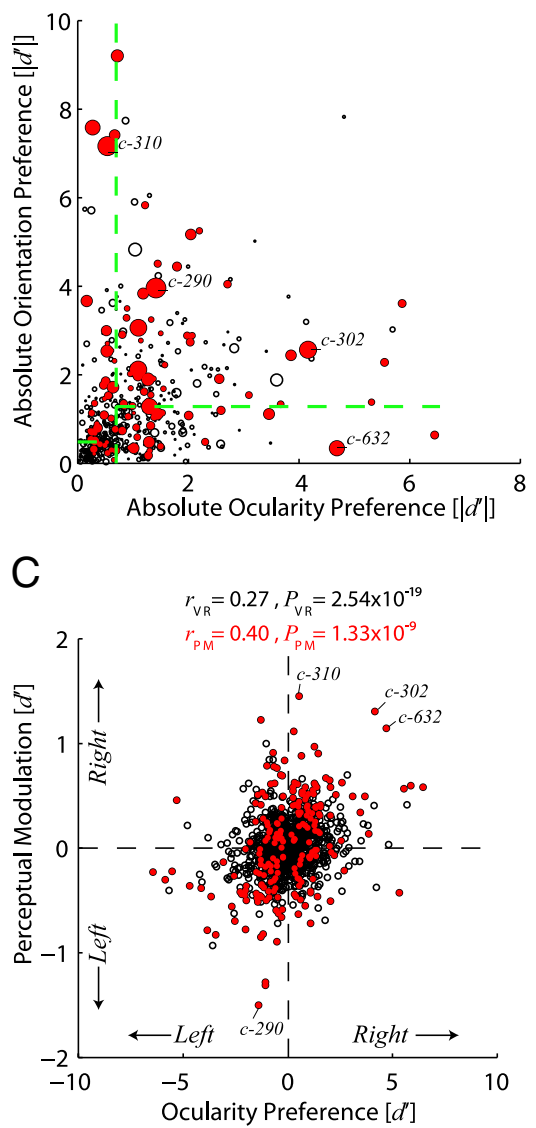

B

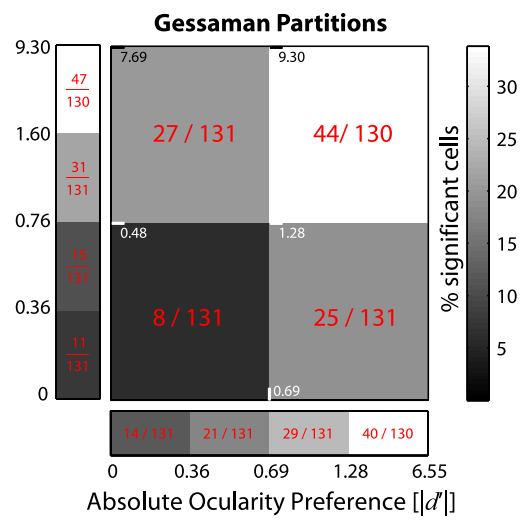

D

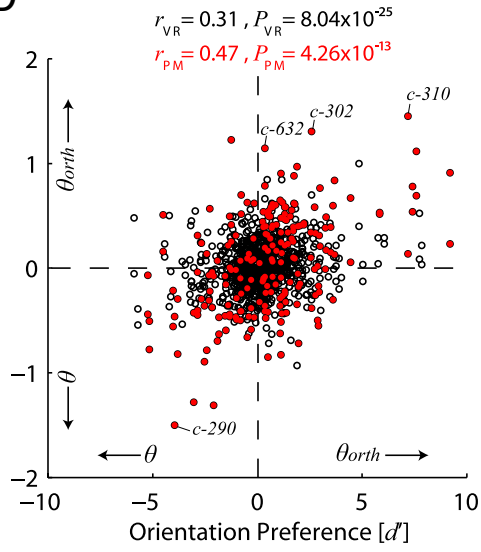

Figure 5. Dependence of perceptual modulation on orientation and ocularity preferences of the neurons. $\boldsymbol{A}$, The distribution of visually responsive (VR) neurons as a function of their ocularity and orientation preferences. The absolute ocularity preference (abscissa) was defined as the absolute $d^{\prime}$ between the responses to left and the right eyes while the absolute orientation preference was defined as the absolute $d^{\prime}$ of the responses to the two different orthogonal orientations (see Materials and Methods for details). Neurons showing significant perceptual modulations (PM) are plotted as red filled circles. The absolute magnitude of the perceptual modulation is represented by the size of the circles. The four neurons presented in Figure $4 B$ are also depicted here by their cell ids. The green dashed-lines show the boundaries of a 4-by-4 Gessaman partitioning $(\boldsymbol{B})$. $\boldsymbol{B}$, The data in $\boldsymbol{A}$ were partitioned using Gessaman partitioning into different groups of four subpopulations containing approximately equal numbers of cells. The main panel partitioned both ocularity and orientation in two while the panels presented on the margins of each axis partitioned the marginal distributions of orientation and ocularity alone, respectively. We then tested whether the underlying numbers of perceptually modulated cells were different in these subpopulations that reflect different ranges of orientation and ocularity preferences, using a $\chi^{2}$ test for homogeneity (see Results for detailed statistics). C, Scatter plot of perceptual modulation as a function of ocularity preference for all visually responsive (VR) cells ( $n=523$, black open circles) and perceptually modulating (PM) cells ( $n=$ 104 , red filled circles). Pearson correlation coefficients were calculated for both populations and are reported on the top of the panel in black and red, respectively. $D$, Scatter plot of perceptual modulation as a function of orientation preference for all visually responsive (VR) cells ( $n=523$, black open circles) and perceptually modulating (PM) cells ( $n=104$, red filled circles). Pearson correlation coefficients were calculated for both populations and are reported on the top of the panel in black and red, respectively.

\section{Results}

\section{Perceptual modulations of single-unit activity}

We recorded neuronal activity from 582 single units from three hemispheres of two awake, behaving monkeys (M. mulatta). Ninety percent $(n=523)$ of these cells were visually responsive (280/303 for D98 and 243/279 for F03). Activity was recorded during binocular flash suppression (BFS), a behavioral paradigm that ensures robust perceptual suppression of a monocular stimulus upon presentation of a second stimulus to the other eye after a delay (Wolfe, 1984; Sheinberg and Logothetis, 1997; Maier et al., 2007). The stimuli were positioned so that they covered the classical receptive fields of the neurons (see Materials and Methods for details).

From a total of 523 visually responsive cells, 371 (71\%) showed significant sensory tuning, which we also refer to as phys- ical preference (Wilcoxon rank-sum test, $\alpha=0.05)$. A substantially smaller number of cells [104 neurons (20\%)] exhibited significant perceptual modulation (i.e., responses modulated with subjective perception, Table 1). Moreover, only a couple of cells (three to four in each monkey) exploited perceptual modulations comparable to their physical preference (see for example Fig. $2 A, D$ ). Instead, most of the perceptually modulated cells showed substantially weaker perceptual modulations compared with their sensory preference during physical alternation (Fig. $2 B, C, E, F)$. Specifically, the magnitude of the average perceptual modulation across these neurons was only 27\% [D98: 26\%, F03: 29\%] compared with the sensory preferences of these cells. Across the population of all cells showing significant physical preference (see method for details) the average magnitude of the perceptual modulation was 15\% [D98: 16\%, F03: 14\%] (Fig. 3). The magnitude of the perceptual effect was also substantially smaller as measured by using the absolute value of $d^{\prime}$ indices [perceptual $d^{\prime}: 0.41 \pm$ 0.02 (SEM), sensory $d^{\prime}: 1.48 \pm 0.09$ (SEM)] (Fig. 4A, inset).

We also found that the sensory and perceptual $d^{\prime}$ indices were significantly positively correlated (Fig. $4 A$, red dots; Pearson $r=0.62, p=1.87 \times 10^{-23}$ ) demonstrating that the strength of the perceptual effect depends on the strength of sensory tuning to the stimuli. Accordingly, the great majority of perceptually modulating cells (84/104) show modulations in the same direction as their physical preference. This is in contrast to other areas like V4 and V5/MT where it was previously shown that half of the perceptually modulated cells showed higher activity during the perception of their nonpreferred stimuli (Logothetis and Schall, 1989; Leopold and Logothetis, 1996). In our study, a very small number of cells $(n=10)$ showed significant perceptual modulations (Wilcoxon rank-sum test, $\alpha=0.05$ ) without showing significant preference in the physical alternation conditions. The rest of the perceptually modulating cells $(n=94)$ showed significant preference during both physical and perceptual alternations (see Table 1).

\section{Monocularity and orientation preference}

We found both monocular and binocular feature-selective cells to be modulated with perception. In addition, neurons that exhibited stronger orientation or ocularity tuning were more likely to show statistically significant perceptual modulations (Fig. 5). We calculated an orientation and an ocularity preference index for each cell, based on their responses to the two orthogonal gratings presented monocularly to each eye (see Materials and 
Methods). The absolute orientation preference index reflects the degree to which the cell preferred one orientation over the orthogonal one. The absolute ocularity preference index measured the degree to which a cell was monocular versus binocular (Fig. 5A). Next, we tested whether the distribution of perceptually modulated cells over these two parameters was different from the distribution of all visually responsive cells. We used a Gessaman partitioning scheme to split the data into four blocks containing approximately equal numbers of cells and performed a $\chi^{2}$ test of homogeneity for these different subpopulations. This type of analysis (Fig. $5 B$ ), revealed a significant difference $\left(\chi^{2}\right.$ test of homogeneity, $p=$ $8.8 \times 10^{-5}$ ) between the percentage of perceptually modulated cells with weak physical tuning (partition near the origin, $8 / 131$ cells) versus all the other three subpopulations that showed stronger physical tuning either in orientation (27/131 cells), ocularity (25/131 cells), or both (44/130 cells). The comparison between the latter three classes did not show a significant difference ( $\chi^{2}$ test of homogeneity, $p=0.07$ ) but the percentage of cells showing strong physical tuning to both orientation and ocularity (44/130 cells) showed a trend to be higher. Gessaman partitioning across orientation alone (see marginal distribution in Fig. $5 B$, ordinate) revealed a gradual increase in the percentages of significantly modulating neurons ( $\chi^{2}$ test of homogeneity, $p=0.007$ ) with increasing orientation preference. Similarly, Gessaman partitioning across ocularity (see marginal distribution in Fig. $5 B$, abscissa) revealed significant gradual increases across ocularity preference $\left(\chi^{2}\right.$ test of homogeneity, $\left.p=1.1 \times 10^{-5}\right)$.

Recently, human fMRI experiments have shown that BOLD activity from V1 voxels can predict the eye-ofpresentation of the perceived stimulus but not its color (Haynes and Rees, 2005). Similarly, we tested whether the strength of perceptual modulations in V1 correlate with the physical orientation preference or the ocularity of the cells recorded. We tested this hypothesis by calculating the correlation coefficients between the amplitude of the perceptual modulations and the physical tuning to ocularity and orientation. We found that perceptual modulations were significantly correlated both with orientation (Fig. 5C; Pearson $r=0.31, p=8.04 \times 10^{-25}$ ) and ocularity (Fig. 5D; Pearson $r=0.27, p=2.54 \times 10^{-19}$ ) preferences of all visually responsive cells. The respective correlation for orientation preference in the population of perceptually modulating cells was (Fig. 5C, red dots; Pearson $r=$ $0.47, p=4.26 \times 10^{-13}$ ) and for ocularity (Fig. $5 D$, red dots; Pearson $r=0.40, p=1.33 \times 10^{-9}$ ).

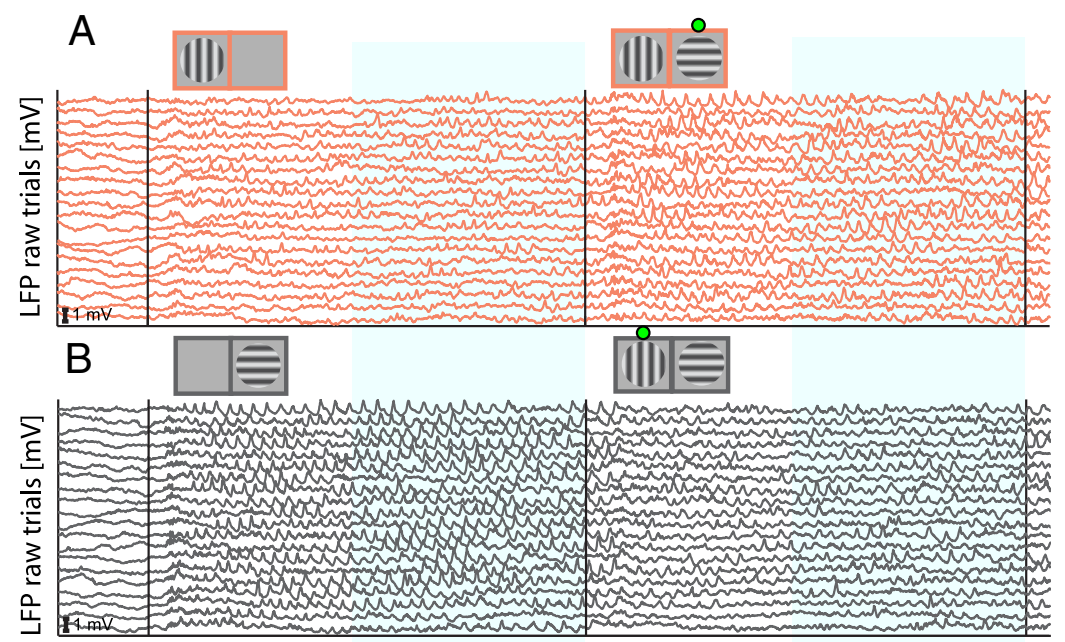

Figure 6. LFP modulations in an example recording site. In $\boldsymbol{A}$ and $\boldsymbol{B}$, we present a sample of 20 single-trial LFP-traces from two same-stimulus different-percept conditions (see Fig. 1). The stimulus sequence is depicted by the diagrams on the top of each panel with the green dots depicting the perceived stimulus during the binocular presentation. C, Power spectra estimates for the two conditions during the monocular stimulus presentation. The power spectra were estimated at a time window between 500 and $1000 \mathrm{~ms}$ (light blue shading) to avoid effects caused by the initial transient response to visual stimulation. A clear increase in gamma-frequencies $(30-100 \mathrm{~Hz})$ and a concurrent decrease in lower LFP frequencies $(<20 \mathrm{~Hz})$ with respect to the spectrum during fixation baseline (black dashed line) can be observed for both conditions. In addition, a clear physical preference for the horizontal grating in the right eye (gray spectrum) versus the vertical grating in the left eye (red spectrum) is noted, especially in the gamma-frequencies. $\boldsymbol{D}$, Similar estimation of spectra performed for the binocular period for which both gratings were present in the two conditions but only one was readily perceived (horizontal grating in the right eye for $A$ condition or vertical grating in the left eye for $\boldsymbol{B}$ condition). Here, the preference for the horizontal grating in the right eye is clearly smaller (red dashed line spectrum versus gray dashed line spectrum). $\boldsymbol{E}$, Time course of LFP modulation in the gamma-frequency range. The LFP traces were bandpass filtered between 30 and $90 \mathrm{~Hz}$ and the average power of the signal is plotted over time. During the monocular presentation there is clear sensory preference to the horizontal grating in the right eye (gray trace) in comparison with the vertical grating in the left eye (light red trace). During the binocular period ( $1000-2000 \mathrm{~ms}$ ), the power shows strong selectivity when the horizontal grating is perceived versus when the vertical is perceived only during the initial $300 \mathrm{~ms}$, after which there is only a very weak difference between the traces of the two conditions. $\boldsymbol{F}$, Similar to $\boldsymbol{E}$, but the LFP traces were bandpass filtered between 4 and $20 \mathrm{~Hz}$. Here both the sensory $(0-1000 \mathrm{~ms})$ and perceptual $(1000-2000 \mathrm{~ms})$ preferences are weak.

\section{Modulations of the local field potentials}

Local field potential signals were acquired from 381 sites recorded from the two monkeys. A typical example of the raw LFP data recorded from a single site is presented in Figure 6, in which an increase in oscillatory activity in the gamma frequency range with a short delay after stimulus onset is evident. In addition, the magnitude of the gamma-band oscillations is significantly different for the two orthogonal monocular gratings (see Fig. 6 for details), indicative of orientation tuning of the gamma-band oscillations (Frien et al., 2000; Berens et al., 2008a,b). In contrast, during the dichoptic presentation representing differences only in the perception of the two stimuli, there was no obvious differ- 
A

Physical Alternation
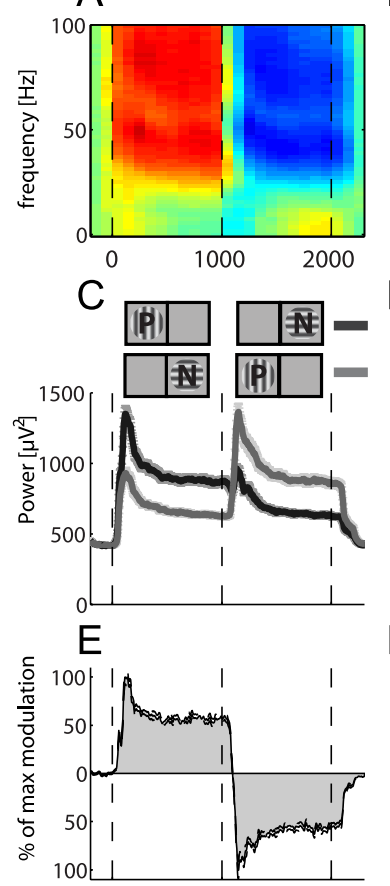

G

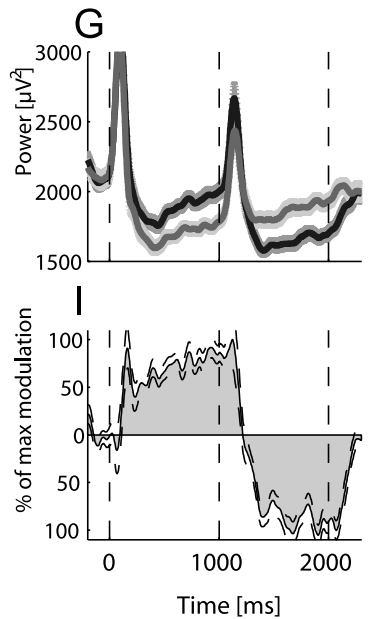

$\mathrm{B}$
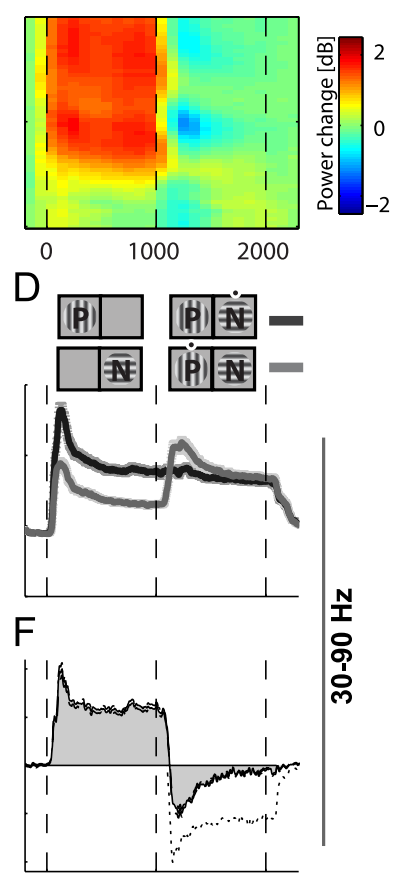

$\mathrm{H}$

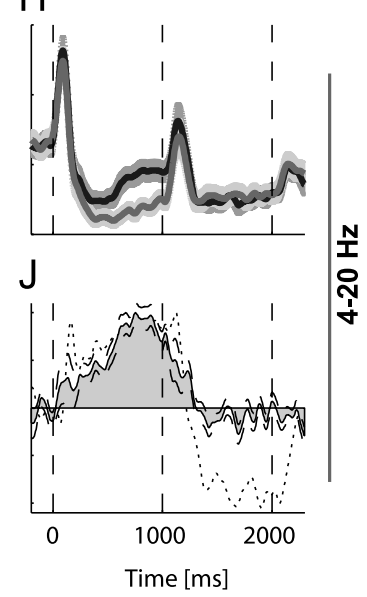

Figure 7. Population averages of the LFP signals for all sites showing physical preferences. Before averaging, conditions were sorted to preferred $(P)$ and nonpreferred $(N)$ according to the responses during the monocular period of presentation. $\boldsymbol{A}$, The average differences in the spectrograms (frequencies $<100 \mathrm{~Hz}$ ) between the preferred and nonpreferred orientations during physical alternation. $\boldsymbol{B}$, The average differences in spectrograms for the same sites under the flash suppression conditions. $\boldsymbol{C}, \boldsymbol{D}, \boldsymbol{G}, \boldsymbol{H}$, Time-domain bandpass filtered averages for the physical alternation and flash suppression conditions for two different frequency bands: the gammaband $(30-90 \mathrm{~Hz})(\boldsymbol{C}, \boldsymbol{D})$, which showed the highest physical preferences $(n=275)$, and the lower frequencies $(4-20 \mathrm{~Hz})(\boldsymbol{G}, \boldsymbol{H})$, which showed weaker preferences $(n=149)$. The sequence of presentation of preferred and nonpreferred is depicted in the diagrams in the upper part of $\boldsymbol{C}$ and $\boldsymbol{D}$ (the black dots denote the perceived stimulus). $\boldsymbol{E}, \boldsymbol{F}, \boldsymbol{I}, \boldsymbol{J}$, Average modulations expressed as differences between preferred and nonpreferred stimuli and shown as a percentage of the maximum modulations during the monocular presentation $(0-1000 \mathrm{~ms})$ for the same two frequency bands: $30-90 \mathrm{~Hz}(\boldsymbol{E}, \boldsymbol{F})$ and $4-20 \mathrm{~Hz}(\boldsymbol{I}, \boldsymbol{J})$. The dotted lines in $\boldsymbol{F}$ and $\boldsymbol{J}$ are copies of the physical alternation in $\boldsymbol{E}$ and $\boldsymbol{I}$ and are presented to provide a means of direct comparison between the flash suppression and the physical alternation modulations.

ence in the raw LFP signals. The same was evident in the power spectral estimates of the LFPs (Fig. 6C,D) and in the band-passed averages in different frequency bands (Fig. $6 E, F$ ).

For each LFP site, we estimated the power spectral densities using the Thomson multitaper method (see Materials and Meth-

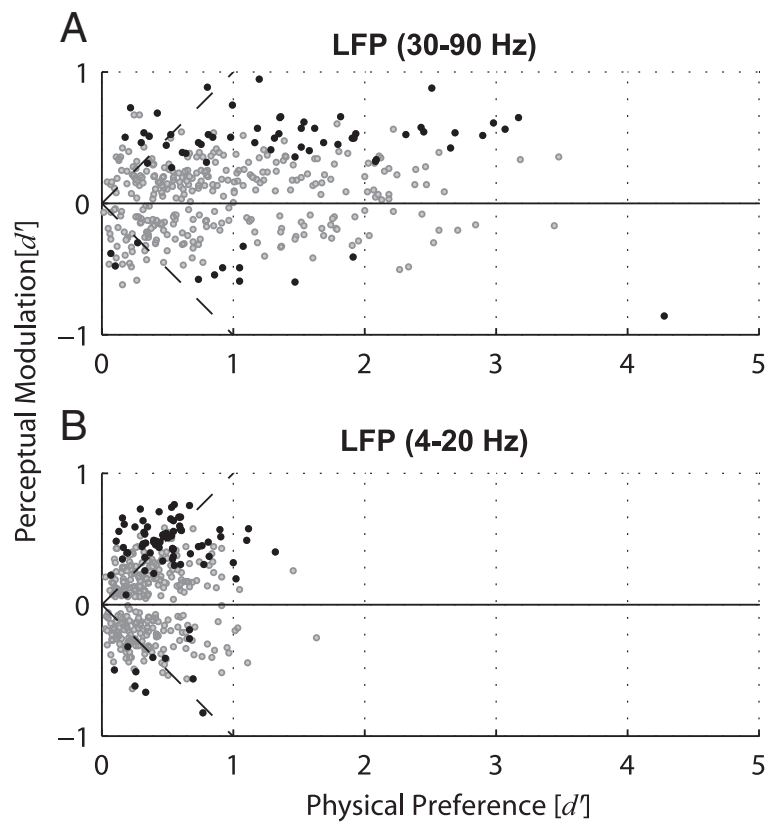

Figure 8. LFP perceptual modulations as a function of physical stimulus preference. $A$, Scatter plot of physical preference versus perceptual modulation of all LFP sites showing significant physical preference in the gamma frequency band $(n=275)$. A positive perceptual modulation index indicates that the response of the LFP site was greater during perception of the preferred grating. A negative perceptual modulation index indicates a higher response during perception of the nonpreferred grating. Dark symbols indicate the sites that showed significant perceptual modulations. $\boldsymbol{B}$, The same as $\boldsymbol{A}$ for the $4-20 \mathrm{~Hz}$ frequency band $(n=149)$.

ods for details) and compared the modulations of different bands for the physical alternation and BFS conditions. The results for two frequency bands are presented in Table 1. We found that $18 \%$ of the visually responsive sites in the gamma frequency band $(30-90 \mathrm{~Hz})$ and $22 \%$ of the visually responsive sites in the frequency band $4-20 \mathrm{~Hz}$ modulated with perception. These values were not significantly different between the two frequency bands and the percentage of perceptually modulated single units $\left(\chi^{2}\right.$ test of homogeneity, $p=0.5$ ).

We quantified the population mean of LFP modulations by using the difference of the average spectrograms of the preferred and nonpreferred orientations (see Materials and Methods). The average power change under the flash suppression conditions was $0.4 \mathrm{~dB}$ (Fig. $7 B$ ) compared with $2.1 \mathrm{~dB}$ under physical alternation conditions (Fig. 7A). We also analyzed perceptual modulations across different frequency bands of the LFP. The average perceptual modulation in the gamma frequency band was weak compared with the modulation during physical alternation (Fig. $7 C, D$ ). On average, the perceptual modulation (during the last $500 \mathrm{~ms}$ ) was $23 \%$ of the modulation of the physical alternation conditions (Fig. $7 E, F$ ). The average perceptual modulations of the lower frequency band (4-20 $\mathrm{Hz}$ ) were also very weak (Fig. $7 \mathrm{G}, H$ ) with an amplitude of $\sim 8 \%$ of the modulation during physical alternation (Fig. 7I,J). The magnitude of the perceptual effect in both frequency bands was also very small as measured by using the $d^{\prime}$ indices of each LFP site (Fig. 8). Notably, the gamma frequency band showed substantially higher sensory preferences compared with the low frequencies (Fig. $8 A, B$ ).

\section{Behavioral responses}

We analyzed the behavioral responses of one animal from a total of 43 sessions [corresponding to 17,230 physical alternation trials and $4025(24.4 \%)$ ] binocular flash suppression/binocular rivalry (BFS/BR) trials) while the animal reported its perception. All 
sessions were acquired after the animals' performance stabilized over 95\% correct responses to presentation of congruent stimuli.

To make sure that BFS worked behaviorally as expected, we analyzed the responses of the animal to both physical alternation and BFS/BR trials. First, we measured the response time and percentage of correct responses of the monkey to physical alternations of two orthogonal gratings (Fig. 9A,C). The monkey consistently reported the correct grating orientation in $96 \%$ of the trials with an average response time of $500 \pm 3 \mathrm{~ms}$ (mean \pm SEM). Then, we calculated the percentage of BFS trials that the monkey reported the new stimulus after the flash. The monkey reported perceiving the newly flashed stimulus in $97 \%$ of the trials (Fig. 9B), demonstrating that, under the stimulation conditions we have used, BFS works in almost all trials. The response time was slightly higher in comparison with physical alternations at $539 \pm 6 \mathrm{~ms}$ (Fig. 9C). We kept the incongruent stimuli on the monitors for up to $5 \mathrm{~s}$ to record the time of spontaneous reversals after the flash. The monkey reported reversals with a mean time of $2057 \pm 51 \mathrm{~ms}$ after the flash, which was longer than the $1000 \mathrm{~ms}$ recorded during the electrophysiological experiments (Fig. 9C). The minimum spontaneous reversal time was $1240 \mathrm{~ms}$. The distribution of spontaneous reversals was fitted by a gamma distribution (Fig. 9C; $r=23.6$ and $\lambda=10.9$ Kolmogorov-Smirnov goodness-of-fit test, $p=0.001$ ).

\section{BFS and neuronal adaptation}

An inherent potential complication of the BFS paradigm is neuronal adaptation. In contrast to $\mathrm{BR}$, the history of stimulation leading to the two alternative percepts is different. This can introduce differences in the level of adaptation in the neuronal populations encoding the competing stimuli. For example, it is expected that when the preferred stimulus of a neuron is presented first then its activity will be reduced further until the end of the trial compared with the case when the preferred stimulus is presented second. This is due to differences in the length of time for adaptation between these two conditions. Therefore, to appreciate the suppressive effect of the second stimulus on the first we should compare a neuron's activity with a condition where the first stimulus is presented alone for the whole duration of the trial. To address this issue, we estimated the level of adaptation for the stimulus presented first across the whole duration of the trial by using an exponential decay function (Fig. 10). We find that the presence of the nonpreferred stimulus (presented second in this case) introduces additional suppression compared with the level of activity predicted by a simple adaptation mechanism (Fig. 10A). Moreover, when the

A

B
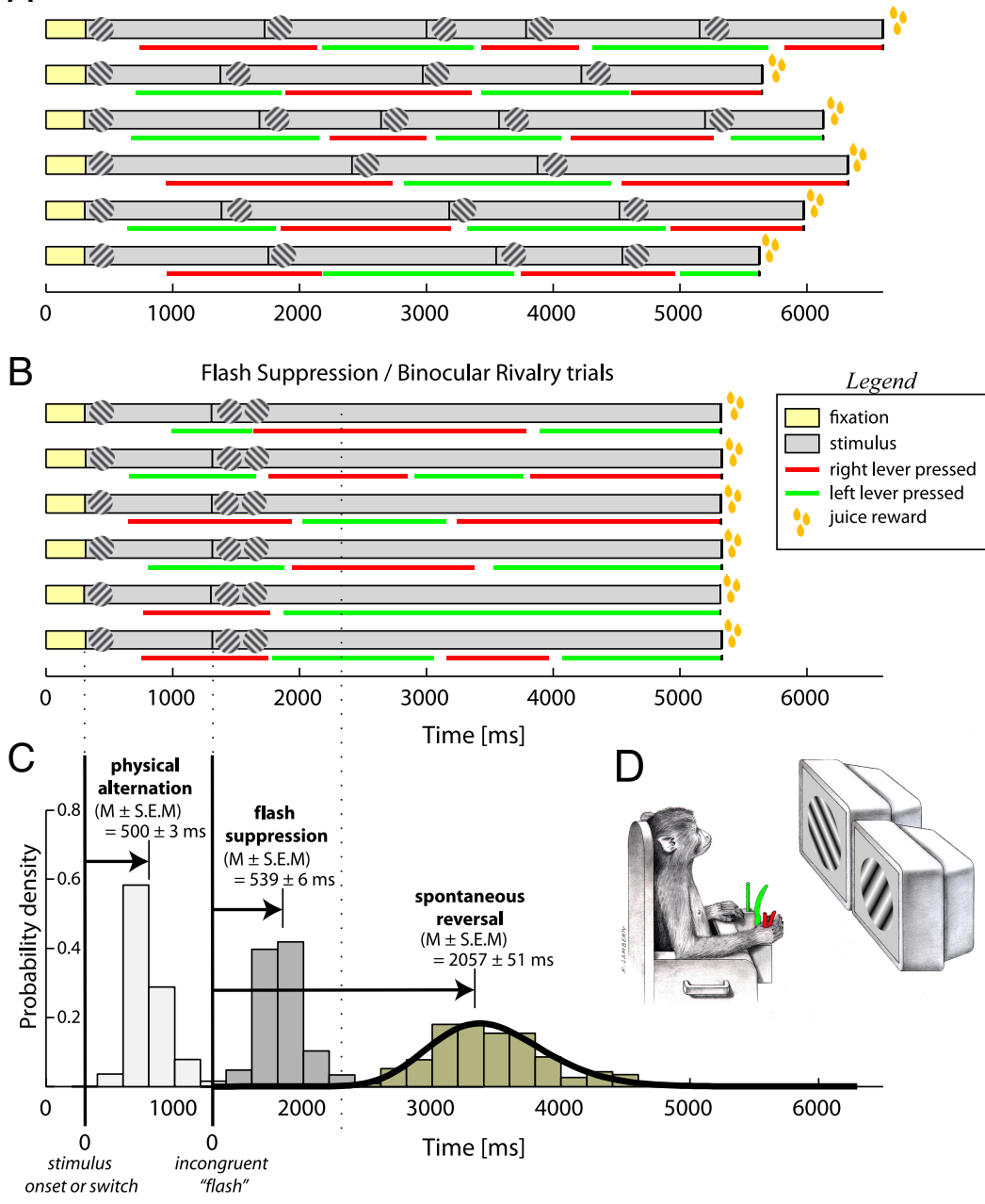

Figure 9. Behavioral responses analysis. A, Demonstration of 6 different physical alternation trials. Each trial started with the monkey fixating a central target for $300 \mathrm{~ms}$ (yellow bar) and then an orientation grating (one of two orthogonal orientations $45^{\circ} / 135^{\circ}$ ) was presented pseudo-randomly on the monitors. The monkey was required to respond by pressing a lever within a time of $1 \mathrm{~s}$. Responses of the monkey are indicated in red and green bars for right and left levers, respectively (see also diagram in $\boldsymbol{D}$ ). Right lever was corresponding to the grating of $45^{\circ}$ orientation while left lever to $135^{\circ}$. The monkey reported consistently the correct orientation in $>95 \%$ of the trials. $B$, Demonstration of 6 different BFS/BR trials (see Materials and Methods presented in one of the two eyes. The monkey responded in the same way and then after the period of $1 \mathrm{~s}$, an orthogonal grating was switched on in the opposite eye. The monkey consistently ( $97 \%$ of trials) reported the newly presented stimulus. The stimuli of response times. On the left the distribution of response times to the presentation of the physical stimulus are presented. The monkey responded with a mean of $500 \pm 3 \mathrm{~ms}$ (mean \pm SEM). His responses to the BFS stimulus were at $539 \pm 6 \mathrm{~ms}$ (mean \pm spontaneous reversals was fitted significantly (Kolmogorov-Smirnov goodness-of-fit test, $p=0.001$ ) by a gamma distribution (see Materials and Methods) with parameters $r=23.6$ and $\lambda=10.9$.

presentation sequence is reversed (i.e., when the preferred appears second) the activity level is also suppressed in comparison with the presentation of the preferred stimulus alone (Fig. $10 \mathrm{~B}$ ). These results, demonstrate that a simple model of activity summation that takes into account adaptation, cannot explain the activity during the binocular incongruent stimulation. Instead, nonlinear interocular interactions are necessary to account for the suppression. Such nonlinear interactions were also present at the level of single neurons. Some cells demonstrated much more suppression than it would be predicted by adaptation (supplemental Fig. $3 D, F, K$, available at www.jneurosci.org as supplemental material) while others exhibited a pronounced enhancement relative to the presentation of the preferred stimu- 
A

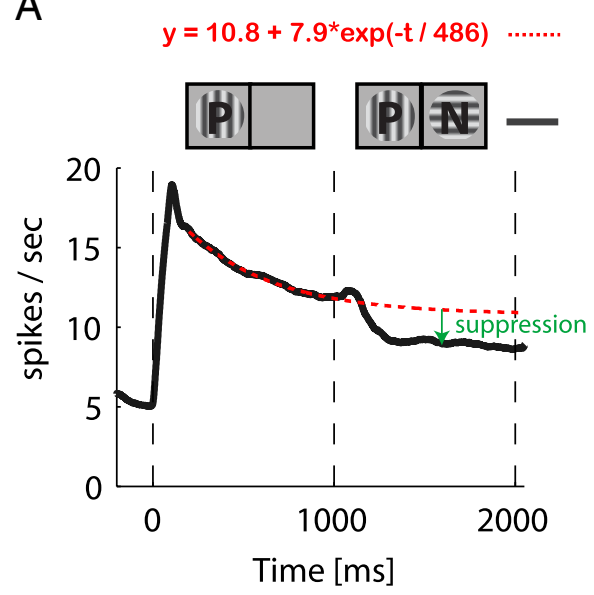

B

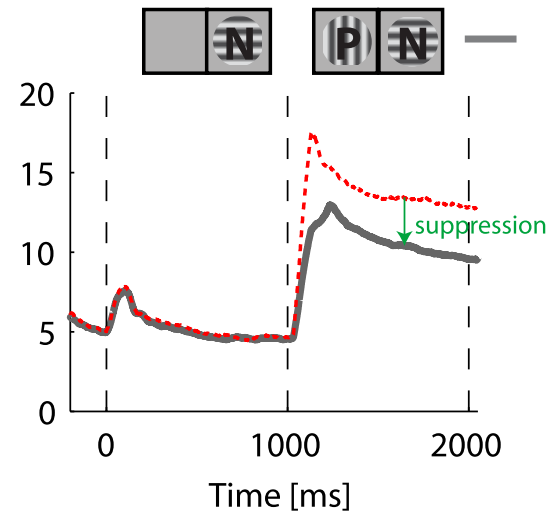

Figure 10. Quantification of the average suppression during incongruent stimulus presentation. In $A$, the suppression caused by the presence and perception of the nonpreferred stimulus ( $N$ ) compared with a modeled (red-dashed line) continuous presentation of the preferred stimulus $(P)$ is shown. $\ln \boldsymbol{B}$, we demonstrate that the presence of the nonpreferred stimulus $(N)$ causes suppression to the preferred $(P)$ even when the preferred stimulus is perceived. The solid black and gray lines are the same as the population averages presented in Figure 3.

lus alone (supplemental Fig. $3 B, C$, available at www.jneurosci. org as supplemental material) that also cannot be explained by adaptation. Importantly the direction of the modulation correlated with perception.

As we have shown above, a trivial model of adaptation cannot explain the responses of V1 neurons during BFS. Nevertheless, this does not exclude more complicated types of adaptation for example affecting only the neurons encoding the perceptually dominant stimulus. Indeed, some models of BR include neuronal adaptation (dependent on the stimulus that is being perceived) as a critical component (Tsuchiya et al., 2006; van Ee, 2009; Kang and Blake, 2010) therefore adaptation likely plays an important role even in studies of classical BR.

\section{Eye movements}

One possible confound that might account for the perceptual modulations we have found is a potential difference in the distribution of eye-movements between the two perceptual conditions. To control for this, we extracted various eye-movement parameters (fixation positions, microsaccade amplitudes, microsaccade directions and microsaccade rates) and compared their distributions between the two different perceptual conditions as we did for the electrophysiological signals (for details see Materials and Methods and supplemental Fig. 1, available at www. jneurosci.org as supplemental material). We did not find a significant difference between the distributions for any of these parameters (see supplemental Fig. 2, available at www.jneurosci. org as supplemental material).

\section{Discussion}

Discovering which aspects of neural activity underlie our subjective percepts and not simply the sensory input impinging upon us has been a question that has fascinated scientists and philosophers for centuries. Specifically, the role that area V1 plays in perception has been a subject of debate (Crick and Koch, 1995; Pollen, 1995). Psychophysical, single-unit and more recently fMRI studies in primates have argued both for and against V1 activity robustly reflecting perception (Blake, 1989; Leopold and Logothetis, 1996; Logothetis et al., 1996; Polonsky et al., 2000; Tong and Engel, 2001). Here we have undertaken a comprehensive study to investi-

gate in detail the extent to which different electrophysiological signals recorded from V1 correlate with perception in awake, behaving monkeys.

Consistent with previous studies the results presented here show that on average the spiking activity of $20 \%$ of V1 neurons follows the perceptual changes of the animal (Leopold and Logothetis, 1996). Furthermore, the cells with stronger physical preference to the stimuli had higher probability to show significant perceptual modulations. Importantly, perceptionrelated modulations were found with equal probability in monocular and binocular orientation-selective neurons. Therefore, we provide for the first time direct evidence at the level of single neurons, supporting the hypothesis that both monocular and binocular processing channels are involved in perception during binocular competition. However, the perception-related modulations in both classes of these neurons are substantially weaker compared with their physical tuning. Average perceptual modulations in different frequency bands of the LFP signals were also week compared with their congruent stimulus selectivities. Thus, to the extent that the magnitude of these modulations provide a robust measure of the involvement of an area in subjective perception, then the primary visual cortex - when compared with other extrastriate areas (Logothetis and Schall, 1989; Leopold and Logothetis, 1996; Sheinberg and Logothetis, 1997) —appears to show substantially weaker modulations.

\section{Binocular versus monocular channels}

Based originally on psychophysical data comparing the persistence of visual after effects under conditions of monoptic and dichoptic stimulation, it has long been speculated that V1 plays a pivotal role in binocular perceptual suppression (Fox and Herrmann, 1967; Blake et al., 1971; Blake and Fox, 1974). Hierarchically, it constitutes the first cortical area receiving information from the two eyes, and monocular neurons in V1 were the speculated candidates for the implementation of suppression through interocular inhibition (Lehky, 1988; Blake, 1989). However, the first electrophysiological evidence in monkeys suggested that in V1/V2 only a small percentage of single neurons were modulated with perception (6/33), and in fact only one of these cells was monocular (Leopold and Logothetis, 1996). In contrast, single-unit recordings from higher visual areas found a larger percentage of neurons correlating with subjective awareness (Logothetis and Schall, 1989; Leopold and Logothetis, 1996; Sheinberg and Logothetis, 1997). These series of studies led to the suggestion that binocular rivalry is probably resolved through competition between stimulus representations at higher visual areas rather than between the monocular channels in V1 (Logothetis, 1998; Leopold and Logothetis, 1999). This idea was also corroborated by psychophysical evidence demonstrating that binocular rivalry can be experienced under conditions whereby dominance and suppression are distributed between the eyes either temporally or spatially (Diaz-Caneja, 1928; Kovács et al., 1996; Logothetis et al., 1996).

Recently, Blake and Logothetis (2002) proposed a hybrid model of rivalry involving a sequence of processes at different 
hierarchical levels of vision, and incorporating possible competition of both ocular and feature-selective channels. Furthermore, additional psychophysical and computational evidence supports the notion that indeed both competition between monocular channels and high-level stimulus interpretations are involved in perception during binocular competition (Dayan, 1998; Brascamp et al., 2007; Ikeda and Morotomi, 2007; Silver and Logothetis, 2007; Bhardwaj et al., 2008). However, electrophysiological evidence supporting this idea has been absent. Here we find that equal percentages of monocular and binocular cells are modulated with perception. In addition, the strength of the perceptual effect correlates with both the orientation and ocularity preferences of the neurons. These results provide the first direct electrophysiological support-at the level of the activity of single cells - for the idea that during BFS, competition does indeed involve mechanisms active across both monocular and binocular neurons. This finding is in agreement with human fMRI evidence for modulations in V1 according to the eye-of-origin of the perceived and suppressed stimuli that led those investigators to reinstate the hypothesis of ocular competition at the level of V1 (Tong and Engel, 2001; Haynes and Rees, 2005) and the lateral geniculate nucleus (Haynes et al., 2005; Wunderlich et al., 2005). Our findings however, illustrate that in addition to eye-of-origin signals, single cells in V1 contain at least equally strong signals for the perceived orientation of the stimulus. In addition, cells tuned to both orientation and ocularity showed a higher tendency to show perceptual modulations. These cells responded differentially for the two different stimulus configurations we have used depending whether the orientation eliciting the stronger response was presented in their preferred eye or the opposite. This further demonstrates that the competition does not happen exclusively for eye-of-origin or orientation signals.

\section{Perceptual modulation of different frequency bands of the local field potential}

In contrast to the electrophysiological studies in monkeys including ours (Leopold and Logothetis, 1996; Gail et al., 2004; Wilke et al., 2006), human fMRI studies have found strong effects of perceptual suppression in the activity of the primary visual cortex (Polonsky et al., 2000). Specifically, the BOLD signal during such perceptual alternations modulates almost as much as when the stimuli are nonambiguously presented separately (physical modulation). The absence of large amplitude modulations in the spiking activity of single cells could potentially reside in a difference in the nature of the two signals (i.e., BOLD and spiking activity of single cells). The modest changes in V1 activity could reflect a modulatory input from higher visual cortices. Such a perception-related modulatory effect could be more pronounced in LFP signals that reflect somatodendritic integrative processes (Mitzdorf, 1987).

In the present study, we analyzed the effects of perceptualtransitions to the different bands of LFP to determine whether indeed LFP signals would reflect perception more robustly. We did not find such evidence. LFP signals during the different perceptual states showed only small modulations compared with the modulations during physical alternation of the stimuli. Our result is partially consistent with a recent study that compared directly single-unit activity, LFP and BOLD activity in behaving-macaques using the generalized flash suppression (GFS) paradigm (Maier et al., 2008). They find different magnitudes of the effect for the electrophysiological and BOLD signals thus confirming the known discrepancy (human fMRI vs monkey electrophysiology) within the same species. However, in agreement with two previously contacted studies (Gail et al., 2004; Wilke et al., 2006), they find that low-frequency LFPs $(<30$
$\mathrm{Hz}$ ) show a modulation ratio substantially larger than the single-unit activity. Moreover, a recent study (Wilke et al., 2009) has demonstrated that low-frequency LFPs in the visual thalamus are critically dependent on the active engagement of the subjects in the task. Specifically, they show that low-frequency LFPs show robust perceptual modulations only if the animals actively report their percepts during GFS. These modulations are eliminated when the animals passively fixate. Therefore, the absence of stronger low-frequency LFP modulations during passive-fixation-BFS we report in our study is entirely consistent with the hypothesis that low-frequency LFPs are reflecting feedback from higher visual areas, which is stronger when the subjects are actively engaged in the task. In addition, this hypothesis can potentially explain the modulations observed in human fMRI studies for which subjects actively reported their percepts. It remains to be shown if perceptual modulations observed in human fMRI can be significantly reduced or abolished when subjects only passively fixate during the presentation of the bistable stimuli. Alternatively, it is possible that differences in the experimental paradigms trigger distinct mechanisms involved in perceptual suppression introducing therefore additional differences in the results (e.g., a potential role of center surround mechanisms in the case of GFS).

Based on the ongoing disagreement between the BOLD results and these classical neurophysiological measures (spikes and power of the LFP in different frequency bands), it is possible that simply measuring the power of the LFP in different frequency bands is not sufficient to capture processes contributing to the modulations of the BOLD signal. Neural activity from multiple sources might be generating field potential changes with inverse signs that add to zero or show minimal changes when recorded using single-point measurements. An example could be spatially disorganized dipoles with minimal or no spatial summation. In addition, anatomy suggests that top-down influences reside mainly in the supragranular layers, pointing to a clear hypothesis for layer specificity.

More detailed studies of the microcircuit organization could reveal whether and how more intricate aspects of activity patterns in $\mathrm{V} 1$ may be more robustly related to the mechanisms of perceptual suppression. For instance, a particular subtype of neurons or specific interactions between cells could robustly encode the percept in V1. An important direction for future research is to dissect and understand in detail the microcircuit mechanisms involved in visual perception.

\section{References}

Abadi RV (1976) Induction masking-a study of some inhibitory interactions during dichoptic viewing. Vision Res 16:269-275.

Attneave F (1971) Multistability in perception. Sci Am 225:63-71.

Baldwin JB, Loop MS, Edwards DJ (1996) Magnitude and time course of interocular suppression is stimulus selective. Invest Ophthalmol Vis Sci 37:3016.

Berens P (2009) CircStat: A MATLAB Toolbox for circular statistics. J Stat Software 31:1-21.

Berens P, Keliris GA, Ecker AS, Logothetis NK, Tolias AS (2008a) Comparing the feature selectivity of the gamma-band of the local field potential and the underlying spiking activity in primate visual cortex. Front Syst Neurosci 2:2.

Berens P, Keliris GA, Ecker AS, Logothetis NK, Tolias AS (2008b) Feature selectivity of the gamma-band of the local field potential in primate primary visual cortex. Front Neurosci 2:199-207.

Bhardwaj R, O’Shea RP, Alais D, Parker A (2008) Probing visual consciousness: rivalry between eyes and images. J Vis 8:2.1-2.13.

Blake R (1989) A neural theory of binocular rivalry. Psychol Rev 96:145-167.

Blake R, Fox R (1974) Adaptation to invisible gratings and the site of binocular rivalry suppression. Nature 249:488-490.

Blake R, Logothetis NK (2002) Visual competition. Nat Rev Neurosci 3: 13-21. 
Blake RR, Fox R, McIntyre C (1971) Stochastic properties of stabilizedimage binocular rivalry alternations. J Exp Psychol 88:327-332.

Blake R, Westendorf D, Fox R (1990) Temporal perturbations of binocular rivalry. Percept Psychophys 48:593-602.

Blake R, Tadin D, Sobel KV, Raissian TA, Chong SC (2006) Strength of early visual adaptation depends on visual awareness. Proc Natl Acad Sci U S A 103:4783-4788.

Brascamp JW, Knapen TH, Kanai R, van Ee R, van den Berg AV (2007) Flash suppression and flash facilitation in binocular rivalry. J Vis 7:12 11-12.

Breese BB (1899) On inhibition. Psychol Rev 3:1-65.

Breese BB (1909) Binocular rivalry. Psychol Rev 16:410-415.

Cogan AI (1987) Human binocular interaction: towards a neural model. Vision Res 27:2125-2139.

Crick F, Koch C (1995) Are we aware of neural activity in primary visual cortex? Nature 375:121-123.

Dayan P (1998) A hierarchical model of binocular rivalry. Neural Comput 10:1119-1135.

Devroye L, Gyorfi L, Lugosi G (1996) A probabilistic theory of pattern recognition. Number 31 in Applications of mathematics (Karatzas I, Yor M, eds). New York: Springer.

Diaz-Caneja E (1928) Sur l'alternance binoculaire. [On binocular alternation]. Ann Oculist 165:721-731.

DuTour M (1760) Discussion d'une question d'optique [Discussion on a question of optics]. In Memoires de Mathematique et de Physique Presentes par Divers Savantes. Paris: Academie des Sciences.

Engbert R, Kliegl R (2003) Microsaccades uncover the orientation of covert attention. Vision Res 43:1035-1045.

Fox R, Herrmann J (1967) Stochastic properties of binocular rivalry alternations. Percept Psychophys 2:432-436.

Frien A, Eckhorn R, Bauer R, Woelbern T, Gabriel A (2000) Fast oscillations display sharper orientation tuning than slower components of the same recordings in striate cortex of the awake monkey. Eur J Neurosci 12: 1453-1465.

Gail A, Brinksmeyer HJ, Eckhorn R (2004) Perception-related modulations of local field potential power and coherence in primary visual cortex of awake monkey during binocular rivalry. Cereb Cortex 14:300-313.

Goense JB, Logothetis NK (2008) Neurophysiology of the BOLD fMRI signal in awake monkeys. Curr Biol 18:631-640.

Haynes JD, Rees G (2005) Predicting the stream of consciousness from activity in human visual cortex. Curr Biol 15:1301-1307.

Haynes JD, Deichmann R, Rees G (2005) Eye-specific effects of binocular rivalry in the human lateral geniculate nucleus. Nature 438:496-499.

Ikeda K, Morotomi T (2007) Alteration of interocular suppression following monocular homogeneous stimulus. Psychologia 50:47-53.

Judge SJ, Richmond BJ, Chu FC (1980) Implantation of magnetic search coils for measurement of eye position: an improved method. Vision Res 20:535-538.

Kang MS, Blake R (2010) What causes alternations in dominance during binocular rivalry? Atten Percept Psychophys 72:179-186.

Kovács I, Papathomas TV, Yang M, Fehér A (1996) When the brain changes its mind: interocular grouping during binocular rivalry. Proc Natl Acad Sci U S A 93:15508-15511.

Kreiman G (2001) On the neuronal activity in the human brain during visual recognition, imagery and binocular rivalry. Ph.D. dissertation, California Institute of Technology.

Kreiman GA, Koch C (1999) Flash suppression: competition between eyes or patterns? Invest Ophthalmol Vis Sci 40:S421.

Kreiman G, Fried I, Koch C (2002) Single-neuron correlates of subjective vision in the human medial temporal lobe. Proc Natl Acad Sci U S A 99:8378-8383.

Lehky SR (1988) An astable multivibrator model of binocular rivalry. Perception 17:215-228.

Leopold DA, Logothetis NK (1996) Activity changes in early visual cortex reflect monkeys' percepts during binocular rivalry. Nature 379:549-553.

Leopold DA, Logothetis NK (1999) Multistable phenomena: changing views in perception. Trends Cogn Sci 3:254-264.

Logothetis NK (1998) Single units and conscious vision. Philos Trans R Soc Lond B Biol Sci 353:1801-1818.

Logothetis NK (1999) Vision: a window on consciousness. Sci Am 281: $69-75$.

Logothetis NK, Schall JD (1989) Neuronal correlates of subjective visual perception. Science 245:761-763.
Logothetis NK, Leopold DA, Sheinberg DL (1996) What is rivalling during binocular rivalry? Nature 380:621-624.

Logothetis NK, Guggenberger H, Peled S, Pauls J (1999) Functional imaging of the monkey brain. Nat Neurosci 2:555-562.

Logothetis NK, Pauls J, Augath M, Trinath T, Oeltermann A (2001) Neurophysiological investigation of the basis of the fMRI signal. Nature 412: $150-157$.

Logothetis N, Merkle H, Augath M, Trinath T, Ugurbil K (2002) Ultra highresolution fMRI in monkeys with implanted RF coils. Neuron 35:227-242.

Macknik SL, Livingstone MS (1998) Neuronal correlates of visibility and invisibility in the primate visual system. Nat Neurosci 1:144-149.

Macknik SL, Martinez-Conde S, Haglund MM (2000) The role of spatiotemporal edges in visibility and visual masking. Proc Natl Acad Sci U S A 97:7556-7560.

Maier A, Logothetis NK, Leopold DA (2007) Context-dependent perceptual modulation of single neurons in primate visual cortex. Proc Natl Acad Sci U S A 104:5620-5625.

Maier A, Wilke M, Aura C, Zhu C, Ye FQ, Leopold DA (2008) Divergence of fMRI and neural signals in V1 during perceptual suppression in the awake monkey. Nat Neurosci 11:1193-1200.

Mitzdorf U (1987) Properties of the evoked potential generators: current source-density analysis of visually evoked potentials in the cat cortex. Int J Neurosci 33:33-59.

Nichols DF, Wilson HR (2009) Effect of transient versus sustained activation on interocular suppression. Vis Res 49:102-114.

Pollen DA (1995) Cortical areas in visual awareness. Nature 377:293-295.

Polonsky A, Blake R, Braun J, Heeger DJ (2000) Neuronal activity in human primary visual cortex correlates with perception during binocular rivalry. Nat Neurosci 3:1153-1159.

Robinson DA (1963) A Method of Measuring Eye Movement Using a Scleral Search Coil in a Magnetic Field. IEEE Trans Biomed Eng 10: 137-145.

Rock I, Hall S, Davis J (1994) Why do ambiguous figures reverse? Acta Psychol (Amst) 87:33-59.

Sengpiel F, Blakemore C (1994) Interocular control of neuronal responsiveness in cat visual cortex. Nature 368:847-850.

Sengpiel F, Blakemore C, Harrad R (1995) Interocular suppression in the primary visual cortex: a possible neural basis of binocular rivalry. Vision Res 35:179-195.

Sheinberg DL, Logothetis NK (1997) The role of temporal cortical areas in perceptual organization. Proc Natl Acad Sci U S A 94:3408-3413.

Silver MA, Logothetis NK (2007) Temporal frequency and contrast tagging bias the type of competition in interocular switch rivalry. Vision Res 47:532-543.

Thomson DJ (1982) Spectrum estimation and harmonic-analysis. Proc IEEE 70:1055-1096.

Tolias AS, Ecker AS, Siapas AG, Hoenselaar A, Keliris GA, Logothetis NK (2007) Recording chronically from the same neurons in awake, behaving primates. J Neurophysiol 98:3780-3790.

Tong F, Engel SA (2001) Interocular rivalry revealed in the human cortical blind-spot representation. Nature 411:195-199.

Tsuchiya N, Koch C, Gilroy LA, Blake R (2006) Depth of interocular suppression associated with continuous flash suppression, flash suppression, and binocular rivalry. J Vis 6:1068-1078.

van Ee R (2009) Stochastic variations in sensory awareness are driven by noisy neuronal adaptation: evidence from serial correlations in perceptual bistability. J Opt Soc Am A 26:2612-2622.

Wheatstone C (1838) On some remarkable, and hitherto unobserved, phenomena of binocular vision. Philos Trans R Soc Lond B Biol Sci 128: 371-394.

Wilke M, Logothetis NK, Leopold DA (2006) Local field potential reflects perceptual suppression in monkey visual cortex. Proc Natl Acad Sci U S A 103:17507-17512.

Wilke M, Mueller KM, Leopold DA (2009) Neural activity in the visual thalamus reflects perceptual suppression. Proc Natl Acad Sci U S A 106: 9465-9470.

Wolfe JM (1984) Reversing ocular dominance and suppression in a single flash. Vision Res 24:471-478.

Wunderlich K, Schneider KA, Kastner S (2005) Neural correlates of binocular rivalry in the human lateral geniculate nucleus. Nat Neurosci 8:1595-1602. 